\title{
A LIKELIHOOD-BASED APPROXIMATE SOLUTION TO THE INCIDENTAL PARAMETER PROBLEM IN DYNAMIC NONLINEAR MODELS WITH MULTIPLE EFFECTS
}

Manuel Arellano and Jinyong Hahn

CEMFI Working Paper No. 0613

December 2006

CEMFI

Casado del Alisal 5; 28014 Madrid

Tel. (34) 914290 551. Fax (34) 914291056

Internet: www.cemfi.es 
CEMFI Working Paper 0613

December 2006

\title{
A LIKELIHOOD-BASED APPROXIMATE SOLUTION TO THE INCIDENTAL PARAMETER PROBLEM IN DYNAMIC NONLINEAR MODELS WITH MULTIPLE EFFECTS
}

\begin{abstract}
We discuss a modified objective function strategy to obtain estimators without bias to order $1 / T$ in nonlinear dynamic panel models with multiple effects. Estimation proceeds from a bias corrected objective function relative to some target infeasible criterion. We consider a determinant based approach for likelihood settings, and a trace based approach, which is not restricted to the likelihood setup. Both approaches depend exclusively on the Hessian and the outer product of the scores of the fixed effects. They produce simple and transparent corrections even in models with multiple effects. We analyze the asymptotic properties of both types of estimators when $n$ and T grow at the same rate, and show that they are asymptotically normal and centered at the truth. Our strategy is to develop a theory for general bias corrected estimating equations, so that we can obtain asymptotic results for a specific bias correction method using the first order conditions.
\end{abstract}

JEL Codes: C23.

Keywords: Nonlinear panel data, fixed effects, bias reduction.

Manuel Arellano

CEMFI

arellano@cemfi.es
Jinyong Hahn

UCLA

hahn@econ.ucla.edu 


\section{Introduction}

There is a body of well understood nonlinear models in econometrics, which are routinely estimated by maximum likelihood or related methods using cross-sectional or time series data. These include, to name a few, discrete choice, conditional volatility, or duration models. In panel data applications of these models, a leading motivation is to exploit the time series variability to allow for heterogeneity in some of the coefficients, which is a powerful way of addressing endogeneity concerns.

Unfortunately, when the time series dimension $T$ is small relative to the cross-sectional dimension $n$, ML estimates of the common parameters or other average effects can be severely biased, specially in dynamic models. This is reflected in asymptotic results such as the fixed- $T$ inconsistency of the ML estimator for some models, or the lack of identification of the model's parameters in a large $n$ fixed- $T$ population for others. Sometimes it is possible to obtain fixed $T$ large $n$ consistent estimators of certain common parameters, based on features of the distribution of the data that do not depend on individual-specific parameters. Nevertheless, situations of this type are more the exception than the rule from the point of view of the needs of applied work.

A useful question is to ask how much heterogeneity can be given empirical content in a particular panel model and data set. One could, for example, expect time series of size 10 to 20 to be statistically informative for up to two or three different coefficients for certain processes. From this perspective, it is natural to choose a population framework that does not rule out the possibility of statistical learning from individual time series in panel data, so that both $T$ and $n$ tend to infinity. If $T$ is statistically informative but much smaller than $n$, as is often the case with micropanels, this should be reflected in the choice of methods of estimation and inference. For example, by seeking estimators with biases of order $1 / T^{2}$ or less as opposed to the standard magnitude of $1 / T$, and asymptotic approximations where $n / T$ or $n / T^{3}$ converge to a constant.

Such is the goal of the recent literature on bias-adjusted estimation methods for nonlinear panel data models with fixed effects. Three different approaches can be distinguished in this literature. One approach is to construct and analytical or numerical bias correction of a fixed effects estimator. Hahn and Newey (2002) considered corrections of this type for static nonlinear panel data models when $n$ and $T$ increase at the same rate, and Hahn and Kuersteiner (2004) provided a similar analysis for dynamic models. A second approach is to consider estimators from bias corrected moment equations. Estimators of this type have been discussed in Woutersen (2002), Arellano (2003), Carro (2004), and Fernandez-Val (2005), amongst others. Finally, a third approach is to consider estimation from a bias corrected objective function relative to some target criterion. Adjustments of this type were discussed in Pace and Salvan (2005) for a generic concentrated likelihood with independent observations, and in Arellano and Hahn (2006) for static nonlinear panel models. ${ }^{1}$

In this paper we consider a modified objective function strategy to obtain estimators without bias

\footnotetext{
${ }^{1}$ See also Arellano and Hahn (2006) for a review of the literature.
} 
to order $1 / T$ in nonlinear dynamic panel models with multiple effects. We consider two approaches to bias correct the objective function, both of which depend on a Hessian term and an outer product of score term, the latter depending on the dynamic dependence of the score. One approach uses a determinant based correction, which we argue later is appropriate in likelihood settings. When the model fully specifies the distribution of the data, it is possible to obtain the expected outer product term and we discuss this possibility. The other approach uses a trace based correction, which we show later is not restricted to the likelihood setup, and is based on a trimmed outer product matrix of the sample score vector. The trace based approach has been independently discussed in a recent paper by Bester and Hansen (2005) as the integral of a bias-corrected moment equation.

Aside from being criterion based, an advantage of these estimators is the great simplicity and transparency of the required corrections by comparison with bias corrections of estimators or moment equations, specially in models with multiple effects. Another benefit of our approach is that bias corrected objective functions can be related to various modifications of the concentrated likelihood suggested in the statistical literature as approximations to conditional or marginal likelihood functions. For example, the determinant based approach is analogous to the Cox and Reid (1987)'s adjusted profile likelihood approach when fixed effects are information orthogonal to common parameters.

We analyze the asymptotic properties of both trace based and determinant based estimators when $n$ and $T$ grow at the same rate, and show that they are asymptotically normal and centered at the truth. Our strategy is to develop a theory for general bias corrected estimating equations, so that we can obtain asymptotic results for a specific bias correction method using the first order conditions.

The paper is organized as follows. Section 2 explains how bias correction of the objective function works. Section 3 presents some examples. Section 4 gives the asymptotic theory. Finally, a brief conclusion is in Section 5. Proofs and technical details are given in the Appendix.

\section{Correcting the Objective Function}

Let the data be denoted by $x_{i t}(t=1, \ldots, T ; i=1, \ldots, n)$. Suppose that we are given a panel data model with a common parameter of interest $\theta_{0}$ and potentially vector-valued individual specific fixed effects $\gamma_{i 0}, i=1, \ldots, n$. We consider a maximization estimator defined by

$$
\left(\widehat{\theta}, \widehat{\gamma}_{1}, \ldots, \widehat{\gamma}_{n}\right) \equiv \underset{\theta, \gamma_{1}, \ldots, \gamma_{n}}{\operatorname{argmax}} \sum_{i=1}^{n} \sum_{t=1}^{T} \psi\left(x_{i t} ; \theta, \gamma_{i}\right)
$$

for some criterion function $\psi(\cdot)$ that does not depend on $T$. Here, $\psi$ is a sensible function in the sense that, if $n$ is fixed, and $T \rightarrow \infty$, the estimator $\left(\widehat{\theta}, \widehat{\gamma}_{1}, \ldots, \widehat{\gamma}_{n}\right)$ is consistent for $\left(\theta_{0}, \gamma_{10}, \ldots, \gamma_{n 0}\right)$.

In a likelihood setup, we assume that $x_{i t}=\left(y_{i t}, y_{i, t-1}, \ldots, y_{i, t-q}\right)$ and

$$
\psi\left(x_{i t} ; \theta, \gamma_{i}\right)=\ln p_{c}\left(y_{i t} \mid y_{i, t-1}, \ldots, y_{i, t-q} ; \theta, \gamma_{i}\right)
$$


where $p_{c}$ denotes the conditional density of $y_{i t} .^{2}$

Letting $\widehat{\gamma}_{i}(\theta) \equiv \operatorname{argmax}_{a} \sum_{t=1}^{T} \psi\left(x_{i t} ; \theta, a\right)$, we can characterize $\widehat{\theta}$ as the estimator that maximizes the concentrated objective function

$$
\widehat{\theta}=\underset{\theta}{\operatorname{argmax}} \frac{1}{n} \sum_{i=1}^{n} \bar{\psi}_{i}\left(\theta, \widehat{\gamma}_{i}(\theta)\right)
$$

where

$$
\bar{\psi}_{i}\left(\theta, \gamma_{i}\right) \equiv \frac{1}{T} \sum_{t=1}^{T} \psi\left(x_{i t} ; \theta, \gamma_{i}\right) .
$$

Now let $\theta_{T}$ be the value that maximizes the limiting expected concentrated objective function for fixed $T$ :

$$
\theta_{T} \equiv \underset{\theta}{\operatorname{argmax}} \lim _{n \rightarrow \infty} \frac{1}{n} \sum_{i=1}^{n} E\left[\bar{\psi}_{i}\left(\theta, \widehat{\gamma}_{i}(\theta)\right)\right] .
$$

Due to the noise in estimating $\widehat{\gamma}_{i}(\theta)$, in general $\theta_{T} \neq \theta_{0}$ (Neyman and Scott (1948)'s incidental parameters problem). This problem would not occur if the quantities $\widehat{\gamma}_{i}(\theta)$ were replaced by $\gamma_{i}(\theta)$ defined $\mathrm{as}^{3}$

$$
\gamma_{i}(\theta) \equiv \underset{c}{\operatorname{argmax}} \lim _{T \rightarrow \infty} \frac{1}{T} \sum_{t=1}^{T} E\left[\psi\left(x_{i t} ; \theta, c\right)\right] .
$$

So we could think of the infeasible concentrated objective function $\sum_{i=1}^{n} \bar{\psi}_{i}\left(\theta, \gamma_{i}(\theta)\right) / n$ as a target criterion and $\sum_{i=1}^{n} \bar{\psi}_{i}\left(\theta, \widehat{\gamma}_{i}(\theta)\right) / n$ as a plug-in estimate with a bias of order $1 / T$. The source of incidental parameter bias is that the concentrated objective function is itself a biased estimate of the target criterion. This suggests maximizing a modified objective function that has no bias up to a certain order in $T$.

For smooth objective functions, the bias in the expected concentrated function at an arbitrary $\theta$ can be usually expanded in orders of magnitude of $T$ :

$$
\lim _{n \rightarrow \infty} E\left[\frac{1}{n} \sum_{i=1}^{n} \bar{\psi}_{i}\left(\theta, \widehat{\gamma}_{i}(\theta)\right)-\frac{1}{n} \sum_{i=1}^{n} \bar{\psi}_{i}\left(\theta, \gamma_{i}(\theta)\right)\right]=\frac{1}{T} B(\theta)+o\left(\frac{1}{T}\right)
$$

for some $B(\theta)$.

A bias corrected concentrated objective function is to plug into the formula for $B(\theta)$ estimators of its unknown components to construct $\widehat{B}(\theta)$, and then obtain an estimator that maximizes the adjusted criterion:

$$
\widetilde{\theta} \equiv \arg \max _{\theta}\left(\frac{1}{n} \sum_{i=1}^{n} \bar{\psi}_{i}\left(\theta, \widehat{\gamma}_{i}(\theta)\right)-\frac{1}{T} \widehat{B}(\theta)\right) .
$$

\footnotetext{
${ }^{2}$ We abstract away from strictly exogenous regressors. For shortness we may write $\psi_{i t}\left(\theta, \gamma_{i}\right)=\psi\left(x_{i t} ; \theta, \gamma_{i}\right)$.

${ }^{3}$ Note that $\gamma_{i}\left(\theta_{0}\right)=\gamma_{i 0}$ and that in the likelihood setup $\gamma_{i}(\theta)$ is fully determined by $\theta$ and the true values, $\theta_{0}$ and $\gamma_{i 0}$.
} 
The resulting estimator removes the leading term of the incidental parameters bias and, unlike $\widehat{\theta}$, it may give correct asymptotic confidence intervals when $T$ grows as fast as $n$.

To see this, consider an expansion for the first order conditions around the truth

$$
\left(-\frac{1}{n} \sum_{i=1}^{n} \frac{\partial^{2}}{\partial \theta \partial \theta^{\prime}} \bar{\psi}_{i}\left(\theta_{0}, \widehat{\gamma}_{i}\left(\theta_{0}\right)\right)\right) \sqrt{n T}\left(\widetilde{\theta}-\theta_{0}\right) \approx \sqrt{n T} \frac{1}{n} \sum_{i=1}^{n} \frac{\partial}{\partial \theta} \bar{\psi}_{i}\left(\theta_{0}, \widehat{\gamma}_{i}\left(\theta_{0}\right)\right)-\sqrt{\frac{n}{T}} \frac{\partial \widehat{B}\left(\theta_{0}\right)}{\partial \theta}
$$

and suppose that $n / T$ tends to a constant, $\sqrt{n T} \sum_{i=1}^{n}(\partial / \partial \theta) \bar{\psi}_{i}\left(\theta_{0}, \gamma_{i}\left(\theta_{0}\right)\right) / n \stackrel{d}{\rightarrow} \mathcal{N}(0, \Omega)$,

$$
\sqrt{n T} \frac{1}{n} \sum_{i=1}^{n} \frac{\partial}{\partial \theta} \bar{\psi}_{i}\left(\theta_{0}, \widehat{\gamma}_{i}\left(\theta_{0}\right)\right)=\sqrt{n T} \frac{1}{n} \sum_{i=1}^{n} \frac{\partial}{\partial \theta} \bar{\psi}_{i}\left(\theta_{0}, \gamma_{i}\left(\theta_{0}\right)\right)+\sqrt{\frac{n}{T}} \frac{\partial B\left(\theta_{0}\right)}{\partial \theta}+o_{p}(1)
$$

and that

$$
\frac{\partial \widehat{B}\left(\theta_{0}\right)}{\partial \theta}=\frac{\partial B\left(\theta_{0}\right)}{\partial \theta}+o_{p}(1)
$$

Thus, also

$$
\sqrt{n T} \frac{1}{n} \sum_{i=1}^{n} \frac{\partial}{\partial \theta} \bar{\psi}_{i}\left(\theta_{0}, \widehat{\gamma}_{i}\left(\theta_{0}\right)\right)-\sqrt{\frac{n}{T}} \frac{\partial \widehat{B}\left(\theta_{0}\right)}{\partial \theta} \stackrel{d}{\rightarrow} \mathcal{N}(0, \Omega)
$$

which suggests that as $n, T \rightarrow \infty, \sqrt{n T}\left(\widetilde{\theta}-\theta_{0}\right)$ is asymptotically normal with zero mean and the same asymptotic variance as the fixed effects estimator. We will give precise conditions for this result to hold.

\subsection{Formulae for the Bias Correction}

Let us introduce the notation:

$$
\begin{aligned}
\bar{V}_{i}\left(\theta, \gamma_{i}\right) & \equiv \frac{\partial \bar{\psi}_{i}\left(\theta, \gamma_{i}\right)}{\partial \gamma_{i}} \\
\bar{H}_{i}(\theta) & \equiv-\lim _{T \rightarrow \infty} E\left[\frac{\partial \bar{V}_{i}\left(\theta, \gamma_{i}(\theta)\right)}{\partial \gamma_{i}^{\prime}}\right] \\
\bar{\Upsilon}_{i}(\theta) & \equiv \lim _{T \rightarrow \infty} T E\left[\bar{V}_{i}\left(\theta, \gamma_{i}(\theta)\right) \bar{V}_{i}\left(\theta, \gamma_{i}(\theta)\right)^{\prime}\right] .
\end{aligned}
$$

A first-order stochastic expansion for an arbitrary fixed $\theta$ gives

$$
\widehat{\gamma}_{i}(\theta)-\gamma_{i}(\theta)=\bar{H}_{i}(\theta)^{-1} \bar{V}_{i}\left(\theta, \gamma_{i}(\theta)\right)+O_{p}\left(\frac{1}{T}\right) .
$$

Next, expanding $\bar{\psi}_{i}\left(\theta, \widehat{\gamma}_{i}(\theta)\right)$ around $\gamma_{i}(\theta)$ for fixed $\theta$ we get

$$
\begin{aligned}
\bar{\psi}_{i}\left(\theta, \widehat{\gamma}_{i}(\theta)\right)-\bar{\psi}_{i}\left(\theta, \gamma_{i}(\theta)\right)= & \bar{V}_{i}\left(\theta, \gamma_{i}(\theta)\right)^{\prime}\left[\widehat{\gamma}_{i}(\theta)-\gamma_{i}(\theta)\right] \\
& -\frac{1}{2}\left[\widehat{\gamma}_{i}(\theta)-\gamma_{i}(\theta)\right]^{\prime} \bar{H}_{i}(\theta)\left[\widehat{\gamma}_{i}(\theta)-\gamma_{i}(\theta)\right]+O_{p}\left(\frac{1}{T^{3 / 2}}\right),
\end{aligned}
$$


and combining the two expansions,

$$
\bar{\psi}_{i}\left(\theta, \widehat{\gamma}_{i}(\theta)\right)-\bar{\psi}_{i}\left(\theta, \gamma_{i}(\theta)\right)=\frac{1}{2} \bar{V}_{i}\left(\theta, \gamma_{i}(\theta)\right)^{\prime} \bar{H}_{i}(\theta)^{-1} \bar{V}_{i}\left(\theta, \gamma_{i}(\theta)\right)+O_{p}\left(\frac{1}{T^{3 / 2}}\right) .
$$

Finally, taking expectations and assuming that the expectations operator and the stochastic order symbols can be interchanged, we obtain

$$
E\left[\bar{\psi}_{i}\left(\theta, \widehat{\gamma}_{i}(\theta)\right)-\bar{\psi}_{i}\left(\theta, \gamma_{i}(\theta)\right)\right]=\frac{1}{T} \beta_{i}(\theta)+O\left(\frac{1}{T^{3 / 2}}\right)
$$

where

$$
\beta_{i}(\theta) \equiv \frac{1}{2} \operatorname{trace}\left[\bar{H}_{i}(\theta)^{-1} \bar{\Upsilon}_{i}(\theta)\right]=\frac{1}{2} \operatorname{trace}\left\{\bar{H}_{i}(\theta) \operatorname{Var}\left(\sqrt{T}\left[\widehat{\gamma}_{i}(\theta)-\gamma_{i}(\theta)\right]\right)\right\} .
$$

In the likelihood setup the information identity is satisfied at the truth so that $\bar{H}_{i}\left(\theta_{0}\right)^{-1} \bar{\Upsilon}_{i}\left(\theta_{0}\right)=$ I. Moreover, $V_{i}\left(x_{i t} ; \theta_{0}, \gamma_{i}\left(\theta_{0}\right)\right)$ is a martingale sequence with the implication that

$$
\bar{\Upsilon}_{i}\left(\theta_{0}\right)=\lim _{T \rightarrow \infty} \frac{1}{T} \sum_{t=1}^{T} E\left[V_{i}\left(x_{i t} ; \theta_{0}, \gamma_{i 0}\right) V_{i}\left(x_{i t} ; \theta_{0}, \gamma_{i 0}\right)^{\prime}\right] \text {. }
$$

When evaluated at other values of $\theta$, the score vector $V_{i}\left(x_{i t} ; \theta, \gamma_{i}(\theta)\right)$ still has zero mean but in general it will be serially correlated:

$$
\bar{\Upsilon}_{i}(\theta)=\sum_{l=-\infty}^{\infty} \bar{\Gamma}_{l}(\theta)
$$

where $\bar{\Gamma}_{l}(\theta)$ denotes the steady-state covariance matrix between $V_{i}\left(x_{i t} ; \theta, \gamma_{i}(\theta)\right)$ and $V_{i}\left(x_{i t-l} ; \theta, \gamma_{i}(\theta)\right)$ :

$$
\bar{\Gamma}_{l}(\theta) \equiv \lim _{T \rightarrow \infty} \frac{1}{T} \sum_{t=l+1}^{T} E\left[V_{i}\left(x_{i t} ; \theta, \gamma_{i}(\theta)\right) V_{i}\left(x_{i t-l} ; \theta, \gamma_{i}(\theta)\right)^{\prime}\right] \quad l>0 .
$$

\subsection{Estimation of the Bias}

An estimator for the bias term in the modified concentrated likelihood (4) can be formed using $\widehat{B}(\theta)=$ $\sum_{i=1}^{n} \widehat{\beta}_{i}(\theta) / n$, where $\widehat{\beta}_{i}(\theta)$ is a sample counterpart of the previous formulae.

Trace Based Approach One possibility is

$$
\widehat{\beta}_{i}(\theta)=\frac{1}{2} \operatorname{trace}\left[H_{i}\left(\theta, \widehat{\gamma}_{i}(\theta)\right)^{-1} \Upsilon_{i}\left(\theta, \widehat{\gamma}_{i}(\theta)\right)\right]
$$

where

$$
\begin{aligned}
H_{i}(\theta, \gamma) & \equiv-\frac{1}{T} \sum_{t=1}^{T} \frac{\partial^{2} \psi_{i t}(\theta, \gamma)}{\partial \gamma \partial \gamma^{\prime}} \\
\Upsilon_{i}(\theta, \gamma) & \equiv \sum_{l=-m}^{m} w_{T, l} \Gamma_{l}(\theta, \gamma) \\
\Gamma_{l}(\theta, \gamma) & \equiv \frac{1}{T} \sum_{t=\max (1, l+1)}^{\min (T, T+l)} \frac{\partial \psi_{i t}(\theta, \gamma)}{\partial \gamma_{i}} \frac{\partial \psi_{i t-l}(\theta, \gamma)}{\partial \gamma_{i}^{\prime}}
\end{aligned}
$$


The quantity $m$ is a bandwidth parameter and $w_{T, l}$ denotes a weight that guarantees positive definiteness of $\Upsilon_{i}(\theta, \gamma)$, e.g., a Bartlett kernel weight such that $w_{T, l}=1-\frac{l}{m+1} \cdot{ }^{4}$ Note that with $m=T-1$ and $w_{T, l}=1, \Upsilon_{i}(\theta, \gamma) \equiv \bar{V}_{i}\left(\theta, \gamma_{i}(\theta)\right) \bar{V}_{i}\left(\theta, \gamma_{i}(\theta)\right)^{\prime}$, so that in such case $\Upsilon_{i}\left(\theta, \widehat{\gamma}_{i}(\theta)\right) \equiv 0$.

The adjustment term $\widehat{\beta}_{i}(\theta)$ does not depend on the likelihood setting, and so it is valid for any fixed effects estimation problem based on the objective function $\sum_{i=1}^{n} \sum_{t=1}^{T} \psi\left(x_{i t} ; \theta, \gamma_{i}\right)$. The tracebased approach can be regarded as an objective-function and estimating equation counterpart to the approach of bias-correction of the estimator in Hahn and Kuersteiner (2004).

Determinant Based Approach In the likelihood setting we can consider a local version of the estimated bias constructed as an expansion of $\widehat{\beta}_{i}(\theta)$ at $\theta_{0}$ using that at the truth $\bar{H}_{i}\left(\theta_{0}\right)^{-1} \bar{\Upsilon}_{i}\left(\theta_{0}\right)=I$. To see this, note that

$$
\widehat{\beta}_{i}(\theta)=\frac{1}{2} \sum_{j=1}^{p}\left[\widehat{\lambda}_{j}(\theta)-1\right]+\frac{1}{2} p=\frac{1}{2} \sum_{j=1}^{p} \ln \widehat{\lambda}_{j}(\theta)+\frac{1}{2} p+O\left(\frac{1}{T}\right)
$$

where $\widehat{\lambda}_{j}(\theta)$ denotes the $j$-th eigenvalue of $H_{i}\left(\theta, \widehat{\gamma}_{i}(\theta)\right)^{-1} \Upsilon_{i}\left(\theta, \widehat{\gamma}_{i}(\theta)\right)$ and $p=\operatorname{dim}(\theta)$. Since $\sum_{j=1}^{p} \ln \widehat{\lambda}_{j}(\theta)=\ln \operatorname{det}\left[H_{i}\left(\theta, \widehat{\gamma}_{i}(\theta)\right)^{-1} \Upsilon_{i}\left(\theta, \widehat{\gamma}_{i}(\theta)\right)\right]$, discarding constants, we can consider the alternative adjustment

$$
\widetilde{\beta}_{i}(\theta)=-\frac{1}{2} \ln \operatorname{det}\left[H_{i}\left(\theta, \widehat{\gamma}_{i}(\theta)\right)\right]+\frac{1}{2} \ln \operatorname{det}\left[\Upsilon_{i}\left(\theta, \widehat{\gamma}_{i}(\theta)\right)\right] .
$$

The resulting modified concentrated likelihood function is

$$
L_{D}(\theta)=\sum_{i=1}^{n} \sum_{t=1}^{T} \psi\left(x_{i t} ; \theta, \widehat{\gamma}_{i}(\theta)\right)+\frac{1}{2} \sum_{i=1}^{n} \ln \operatorname{det}\left[H_{i}\left(\theta, \widehat{\gamma}_{i}(\theta)\right)\right]-\frac{1}{2} \sum_{i=1}^{n} \ln \operatorname{det}\left[\Upsilon_{i}\left(\theta, \widehat{\gamma}_{i}(\theta)\right)\right]
$$

where $\psi\left(x_{i t} ; \theta, \gamma_{i}\right)=\ln p_{c}\left(y_{i t} \mid y_{i, t-1}, \ldots, y_{i, t-q} ; \theta, \gamma_{i}\right)$.

The criterion $L_{D}(\theta)$ is a multivariate and dynamic version of the adjusted concentrated likelihood considered by DiCiccio and Stern (1993), and DiCiccio, Martin, Stern, and Young (1996).

Using the arguments in Pace and Salvan (2005), it can be related to the adjusted profile likelihood considered by Cox and Reid (1987) as an approximation to the likelihood conditioned on the ML estimates of the fixed effects. In a model with independent observations, Ferguson, Reid, and Cox (1991) showed that such a modification led to bias reduction when the nuisance parameters were information orthogonal to the parameters of interest.

In our context, the Cox-Reid approach maximizes

$$
L_{C R}(\theta)=\sum_{i=1}^{n} \sum_{t=1}^{T} \psi\left(x_{i t} ; \theta, \widehat{\gamma}_{i}(\theta)\right)-\frac{1}{2} \sum_{i=1}^{n} \ln \operatorname{det}\left[H_{i}\left(\theta, \widehat{\gamma}_{i}(\theta)\right)\right],
$$

\footnotetext{
${ }^{4}$ For simplicity of exposition, we will assume that the $w_{T, l}$ are indeed Bartlett weights throughout the rest of the paper.
} 
and the connection with $L_{D}(\theta)$ can be expressed as

$$
L_{D}(\theta)=L_{C R}(\theta)-\frac{1}{2} \sum_{i=1}^{n} \ln \operatorname{det} \widehat{\operatorname{Var}}\left[\sqrt{n T}\left(\widehat{\gamma}_{i}(\theta)-\gamma_{i}(\theta)\right)\right]
$$

where the variance term is given by the sandwich formula

$$
\widehat{\operatorname{Var}}\left[\sqrt{n T}\left(\widehat{\gamma}_{i}(\theta)-\gamma_{i}(\theta)\right)\right]=\left[H_{i}\left(\theta, \widehat{\gamma}_{i}(\theta)\right)\right]^{-1} \Upsilon_{i}\left(\theta, \widehat{\gamma}_{i}(\theta)\right)\left[H_{i}\left(\theta, \widehat{\gamma}_{i}(\theta)\right)\right]^{-1}
$$

The conclusion is that $L_{D}(\theta)$ can be regarded as a generalized Cox-Reid function with an additional term to account for non-orthogonality. Under orthogonality the extra term is not needed because the variance of $\widehat{\gamma}_{i}(\theta)$ does not change much with $\theta$.

Determinant Approach Using Expected Quantities In the likelihood setting, an expected outer product function can be calculated for given values of $\left(\theta, \gamma_{i}\right)$ and $\left(\theta_{0}, \gamma_{i 0}\right)$ analytically or numerically, because the density of the data is available. Specifically, we may consider

$$
\Upsilon_{T i}\left(\theta, \gamma ; \theta_{0}, \gamma_{i 0}\right) \equiv \sum_{l=-m}^{m} w_{T, l} \Gamma_{T l}\left(\theta, \gamma ; \theta_{0}, \gamma_{i 0}\right)
$$

where, for $l>0$, we have

$$
\Gamma_{T l}\left(\theta, \gamma ; \theta_{0}, \gamma_{i 0}\right)=\frac{1}{T-l} \sum_{t=l+1}^{T} E_{\theta_{0}, \gamma_{i 0}}\left[V_{i}\left(x_{i t} ; \theta, \gamma\right) V_{i}\left(x_{i t-l} ; \theta, \gamma\right)^{\prime}\right]
$$

Alternatively, a centered covariance could be calculated:

$$
\Gamma_{T l}^{*}\left(\theta, \gamma ; \theta_{0}, \gamma_{i 0}\right)=\Gamma_{T l}\left(\theta, \gamma ; \theta_{0}, \gamma_{i 0}\right)-\mu_{T 0}\left(\theta, \gamma ; \theta_{0}, \gamma_{i 0}\right) \mu_{T l}\left(\theta, \gamma ; \theta_{0}, \gamma_{i 0}\right)^{\prime}
$$

where $\mu_{T l}\left(\theta, \gamma ; \theta_{0}, \gamma_{i 0}\right)=(T-l)^{-1} \sum_{t=l+1}^{T} E_{\theta_{0}, \gamma_{i 0}}\left[V_{i}\left(x_{i t-l} ; \theta, \gamma\right)\right]$. Note that when evaluated at $\gamma=$ $\gamma_{i}(\theta)$ for arbitrary $\theta$ we have $\mu_{T l}\left(\theta, \gamma_{i}(\theta) ; \theta_{0}, \gamma_{i 0}\right)=0$, so that centered and non-centered quantities coincide.

This leads to an alternative modified concentrated likelihood of the form

$$
L_{E D}(\theta ; \widehat{\theta})=\sum_{i=1}^{n} \sum_{t=1}^{T} \psi\left(x_{i t} ; \theta, \widehat{\gamma}_{i}(\theta)\right)+\frac{1}{2} \sum_{i=1}^{n} \ln \operatorname{det} H_{i}\left(\theta, \widehat{\gamma}_{i}(\theta)\right)-\frac{1}{2} \sum_{i=1}^{n} \ln \operatorname{det} \Upsilon_{T i}\left(\theta, \widehat{\gamma}_{i}(\theta) ; \widehat{\theta}, \widehat{\gamma}_{i}(\widehat{\theta})\right)
$$

Iterated Adjusted Likelihood Estimation An undesirable feature of the estimator $\widehat{\theta}_{1}=$ $\arg \max _{\theta} L_{E D}(\theta ; \widehat{\theta})$ is its dependence on $\widehat{\theta}$, which may have a large bias. This problem can be avoided by considering an iterative procedure. That is, once we have $\widehat{\theta}_{1}$, we use it to evaluate the expectations required in calculating a new estimate. Pursuing the iteration

$$
\widehat{\theta}_{K}=\arg \max _{\theta} L_{E D}\left(\theta ; \widehat{\theta}_{K-1}\right)
$$


until convergence, we obtain an estimator $\widehat{\theta}_{\infty}$ that solves

$$
S_{E D}\left(\widehat{\theta}_{\infty} ; \widehat{\theta}_{\infty}\right)=0
$$

where $S_{E D}\left(\theta ; \theta_{*}\right)$ denotes the score of $L_{E D}\left(\theta ; \theta_{*}\right)$ for fixed $\theta_{*}$. Note that, in contrast with the iterated procedure, a continuously updated method will not work in this case (that is, maximizing a criterion of the form $\left.L_{E D}(\theta ; \theta)\right)$.

Discussion Both likelihood and pseudo likelihood settings are important in applications. For example, there are nonlinear likelihood models whose parameters are no longer interpretable when the likelihood is only regarded as a pseudo likelihood.

In a likelihood situation it seems natural to use the determinant form of the correction, but also an expectation based estimate of the outer product term, specially if an analytical calculation is available, hence avoiding semiparametric kernel estimation. However, if expectations need to be evaluated by simulation, the conceptual advantage of the expectation-based adjustment is less clear, because the number of simulations to be chosen is an issue.

In contrast, in a pseudo likelihood or incomplete model setting it is natural to use the trace form of the correction and a kernel-based estimate of $\bar{\Upsilon}_{i}(\theta)$, which is the only possibility available.

\section{Examples}

We consider three examples. The first one is static and linear, but illustrates the differences between the two approaches in a familiar context. The second is a conditional volatility model, and the last one is a dynamic binary choice formulation.

Example 1 Consider a simple multivariate model for an unconditional covariance structure with heterogeneous means, where

$$
\psi\left(x_{i t} ; \theta, \gamma_{i}\right)=C-\frac{1}{2} \ln \operatorname{det} \Omega(\theta)-\frac{1}{2}\left(x_{i t}-\gamma_{i}\right)^{\prime} \Omega(\theta)^{-1}\left(x_{i t}-\gamma_{i}\right) .
$$

If $\Omega(\theta)$ is unrestricted then $\theta=\operatorname{vech}[\Omega(\theta)]$. We have $\widehat{\gamma}_{i}(\theta)=\bar{x}_{i}$ and

$$
\begin{aligned}
\frac{\partial \psi\left(x_{i t} ; \theta, \gamma_{i}\right)}{\partial \gamma_{i}} & =\Omega(\theta)^{-1}\left(x_{i t}-\gamma_{i}\right), \quad \frac{\partial^{2} \psi\left(x_{i t} ; \theta, \gamma_{i}\right)}{\partial \gamma_{i} \partial \gamma_{i}^{\prime}}=-\Omega(\theta)^{-1} \\
H_{i}(\theta, \gamma) & \equiv-\frac{1}{T} \sum_{t=1}^{T} \frac{\partial^{2} \psi_{i t}(\theta, \gamma)}{\partial \gamma \partial \gamma^{\prime}}=\Omega(\theta)^{-1} \\
\Upsilon_{i}\left(\theta, \widehat{\gamma}_{i}(\theta)\right) & \equiv \sum_{l=-m}^{m} w_{T, l} \Gamma_{l}\left(\theta, \widehat{\gamma}_{i}(\theta)\right) \\
\Gamma_{l}\left(\theta, \widehat{\gamma}_{i}(\theta)\right) & \equiv \Omega(\theta)^{-1}\left[\frac{1}{T} \sum_{t=\max (1, l+1)}^{\min (T, T+l)}\left(x_{i t}-\bar{x}_{i}\right)\left(x_{i t-l}-\bar{x}_{i}\right)^{\prime}\right] \Omega(\theta)^{-1} .
\end{aligned}
$$


The determinant approach with $m=0$ gives

$$
\begin{aligned}
L_{D}(\theta)= & C-\frac{n T}{2} \ln \operatorname{det} \Omega(\theta)-\frac{1}{2} \sum_{i=1}^{n} \sum_{t=1}^{T}\left(x_{i t}-\bar{x}_{i}\right)^{\prime} \Omega(\theta)^{-1}\left(x_{i t}-\bar{x}_{i}\right) \\
& +\frac{n}{2} \ln \operatorname{det}\left[\Omega(\theta)^{-1}\right]-\frac{1}{2} \ln \operatorname{det}\left(\Omega(\theta)^{-1} \frac{1}{T} \sum_{i=1}^{n} \sum_{t=1}^{T}\left(x_{i t}-\bar{x}_{i}\right)\left(x_{i t}-\bar{x}_{i}\right)^{\prime} \Omega(\theta)^{-1}\right) .
\end{aligned}
$$

Finally, collecting terms and discarding constants we get

$$
L_{D}(\theta)=C-\frac{n(T-1)}{2} \ln \operatorname{det} \Omega(\theta)-\frac{n T}{2} \operatorname{trace}\left[\Omega(\theta)^{-1} \widehat{\Omega}\right]
$$

where $\widehat{\Omega}$ is the unrestricted fixed effects estimate:

$$
\widehat{\Omega}=\frac{1}{n T} \sum_{i=1}^{n} \sum_{t=1}^{T}\left(x_{i t}-\bar{x}_{i}\right)\left(x_{i t}-\bar{x}_{i}\right)^{\prime} .
$$

Thus, the information adjustment performs the required degrees of freedom correction (i.e. the corrected unrestricted estimate is $\widetilde{\Omega}=\frac{T}{T-1} \widehat{\Omega}$ ).

The trace-based approach should provide bias reduction in the presence of neglected serial correlation. It gives

$$
\widehat{\beta}_{i}(\theta)=\frac{1}{2} \operatorname{trace}\left[\widetilde{\Gamma}_{i} \Omega(\theta)^{-1}\right]
$$

where

$$
\widetilde{\Gamma}_{i}=\frac{1}{T} \sum_{l=-m}^{m} w_{T, l} \sum_{t=\max (1, l+1)}^{\min (T, T+l)}\left(x_{i t}-\bar{x}_{i}\right)\left(x_{i t-l}-\bar{x}_{i}\right)^{\prime} .
$$

Letting $\widetilde{\Gamma}=n^{-1} \sum_{i=1}^{n} \widetilde{\Gamma}_{i}$, we obtain

$$
L_{T R}(\theta)=C-\frac{n T}{2} \ln \operatorname{det} \Omega(\theta)-\frac{n T}{2} \operatorname{trace}\left[\Omega(\theta)^{-1} \widehat{\Omega}\right]-\frac{n}{2} \operatorname{trace}\left[\Omega(\theta)^{-1} \widetilde{\Gamma}\right] .
$$

Note that with $m=0, \widetilde{\Gamma}=\widehat{\Omega}$, so that in this case the corrected unrestricted estimate is $\widetilde{\Omega}_{T R}=$ $\frac{T+1}{T} \widehat{\Omega}$, which removes the bias of order $T^{-1}$, but is not fully unbiased. In general, the trace-based unrestricted estimate is given by

$$
\widetilde{\Omega}_{T R}=\widehat{\Omega}+\frac{1}{T} \widetilde{\Gamma}
$$

Example 2 The next example is a heteroskedastic autoregressive model with two fixed effects, one in the conditional mean and another in the conditional variance. Letting $\theta=\left(\theta_{1}, \theta_{2}\right)$ and $\gamma_{i}=$ $\left(\gamma_{1 i}, \gamma_{2 i}\right)$, we have

$$
\psi\left(x_{i t} ; \theta, \gamma_{i}\right)=-\frac{1}{2} \ln h\left(y_{i t-1}, \gamma_{2 i}\right)-\frac{1}{2} \frac{\left(y_{i t}-\theta_{1} y_{i t-1}-\gamma_{1 i}\right)^{2}}{h\left(y_{i t-1}, \gamma_{2 i}\right)}
$$


where

$$
h\left(y_{i t-1}, \gamma_{2 i}\right)=\left(\gamma_{2 i}+\theta_{2} y_{i t-1}\right)^{2} .
$$

A model of this type, but with an exponential ARCH formulation of the conditional variance, is developed in Hospido (2006), where some of the estimators considered in this paper, as well as simulationbased alternatives, are implemented and applied to study individual wage dynamics.

Example 3 A third example is an autoregressive binary formulation of the form

$$
\psi\left(x_{i t} ; \theta, \gamma_{i}\right)=y_{i t} \ln \Lambda\left(\gamma_{1 i}+\gamma_{2 i} y_{i t-1}+\theta y_{i t-2}\right)+\left(1-y_{i t}\right) \ln \left[1-\Lambda\left(\gamma_{1 i}+\gamma_{2 i} y_{i t-1}+\theta y_{i t-2}\right)\right]
$$

where $\Lambda(r)$ is the logit or probit $c d f$.

This model was suggested in Chamberlain (1985) as a framework for testing duration dependence from binary panel data, by testing the restriction $\theta=0$. Chamberlain showed that, in the absence of exogenous variables, a simple fixed- $T$ consistent estimator for $\theta$ is available for the logistic specification of this model. A random effects formulation of a model of this type has been recently applied by Card and Hyslop (2005) to study the effects of earnings subsidies on welfare participation.

\section{Asymptotic Theory}

We first consider general conditions for a bias corrected estimating equation to deliver an asymptotic normality theorem for the estimation error centered at the truth.

Notation 1 We use the following additional notation throughout:

$$
\begin{aligned}
& U_{i}\left(x_{i t} ; \theta, \gamma_{i}\right) \equiv \frac{\partial \psi\left(x_{i t} ; \theta, \gamma_{i}\right)}{\partial \theta}-\rho_{i 0} \cdot \frac{\partial \psi\left(x_{i t} ; \theta, \gamma_{i}\right)}{\partial \gamma_{i}}, \quad V_{i}\left(x_{i t} ; \theta, \gamma_{i}\right) \equiv \frac{\partial \psi\left(x_{i t} ; \theta, \gamma_{i}\right)}{\partial \gamma_{i}}, \\
& \rho_{i 0} \equiv E\left[\frac{\partial^{2} \psi\left(x_{i t} ; \theta_{0}, \gamma_{i 0}\right)}{\partial \theta \partial \gamma_{i}^{\prime}}\right]\left(E\left[\frac{\partial^{2} \psi\left(x_{i t} ; \theta_{0}, \gamma_{i 0}\right)}{\partial \gamma_{i} \partial \gamma_{i}^{\prime}}\right]\right)^{-1}, \quad \mathcal{I}_{i} \equiv-E\left[\frac{\partial U_{i}\left(x_{i t} ; \theta_{0}, \gamma_{i 0}\right)}{\partial \theta^{\prime}}\right], \\
& \widetilde{V}_{i t} \equiv-\left(E\left[\frac{\partial V_{i}}{\partial \gamma_{i}^{\prime}}\right]\right)^{-1} V_{i t} .
\end{aligned}
$$

For simplicity of notation, we will occasionally write $U_{i t} \equiv U_{i}\left(x_{i t} ; \theta_{0}, \gamma_{i 0}\right)$ and $V_{i t} \equiv V_{i}\left(x_{i t} ; \theta_{0}, \gamma_{i 0}\right)$. We will denote by $U_{i t}^{\gamma_{i}} \equiv \partial U_{i t} / \partial \gamma_{i}^{\prime}$ and $U_{i t}^{\gamma_{i} \gamma_{i}} \equiv \partial^{2} U_{i t} /\left(\partial \gamma_{i}^{\prime} \otimes \partial \gamma_{i}^{\prime}\right)$ the first and second derivatives of $U_{i t}$ with respect to $\gamma_{i}$. Likewise, we will denote by $V_{i t}^{\gamma_{i}}$ the derivative $\partial V_{i t} / \partial \gamma_{i}^{\prime}$ of $V_{i t}$ with respect to $\gamma_{i}$.

Using this notation, we can characterize $\widehat{\theta}$ as the solution to the first order condition

$$
0=\sum_{i=1}^{n} \sum_{t=1}^{T} U_{i}\left(x_{i t} ; \widehat{\theta}, \widehat{\gamma}_{i}(\widehat{\theta})\right) .
$$


The normalized score $\frac{1}{n T} \sum_{i=1}^{n} \sum_{t=1}^{T} U\left(x_{i t} ; \theta_{0}, \widehat{\gamma}_{i}\left(\theta_{0}\right)\right)$ has an asymptotic bias, which renders the fixed effects estimator $\widehat{\theta}$ biased. The asymptotic bias of the normalized score can be $\operatorname{shown}^{5}$ to be equal to $\frac{1}{T}$ times $\Psi_{0}\left(\theta_{0},\left\{\gamma_{10}, \gamma_{20}, \ldots\right\}\right)$, where

$$
\begin{aligned}
\Psi_{0}\left(\theta_{0},\left\{\gamma_{10}, \gamma_{20}, \ldots\right\}\right)= & \operatorname{plim} \frac{1}{n} \sum_{i=1}^{n}\left(\frac{1}{\sqrt{T}} \sum_{t=1}^{T} U_{i t}^{\gamma_{i}}\right)\left(\frac{1}{\sqrt{T}} \sum_{t=1}^{T} \widetilde{V}_{i t}\right) \\
& +\operatorname{plim} \frac{1}{2} \frac{1}{n} \sum_{i=1}^{n} E\left[U_{i}^{\gamma_{i} \gamma_{i}}\right]\left[\left(\frac{1}{\sqrt{T}} \sum_{t=1}^{T} \widetilde{V}_{i t}\right) \otimes\left(\frac{1}{\sqrt{T}} \sum_{t=1}^{T} \widetilde{V}_{i t}\right)\right] .
\end{aligned}
$$

Note that $\gamma_{i 0} \equiv \operatorname{argmax}_{c} E\left[\psi\left(x_{i t} ; \theta_{0}, c\right)\right]$. Therefore, using $\gamma_{i}(\theta) \equiv \operatorname{argmax}_{c} E\left[\psi\left(x_{i t} ; \theta, c\right)\right]$, we can write

$$
\Psi_{0}\left(\theta_{0},\left\{\gamma_{10}, \gamma_{20}, \ldots\right\}\right)=\Psi_{0}\left(\theta_{0},\left\{\gamma_{1}\left(\theta_{0}\right), \gamma_{2}\left(\theta_{0}\right), \ldots\right\}\right)
$$

which can be regarded as a function in $\theta_{0}$. Such function will be written as $\Psi_{0}(\theta)$ without loss of generality. We will approximate it by $\Psi_{n}\left(\theta_{0}\right) \equiv \Psi_{0}\left(\theta_{0},\left\{\widehat{\gamma}_{1}\left(\theta_{0}\right), \widehat{\gamma}_{2}\left(\theta_{0}\right), \ldots\right\}\right)$. Letting $S_{n}\left(\theta_{0}\right)$ denote some sample counter-part of $\Psi_{n}\left(\theta_{0}\right)$, we may consider solving

$$
0=\frac{1}{n T} \sum_{i=1}^{n} \sum_{t=1}^{T} U\left(x_{i t} ; \widetilde{\theta}, \widehat{\gamma}_{i}(\widetilde{\theta})\right)-\frac{1}{T} S_{n}(\widetilde{\theta})
$$

instead. We will assume that there exists some $B_{n}$ such that $S_{n}(\theta)=\partial B_{n}(\theta) / \partial \theta$, in which case our estimator $\widetilde{\theta}$ can be understood as a solution to

$$
\underset{\theta, \gamma_{1}, \ldots, \gamma_{n}}{\operatorname{argmax}} \frac{1}{n T} \sum_{i=1}^{n} \sum_{t=1}^{T} \psi\left(x_{i t} ; \theta, \gamma_{i}\right)-\frac{1}{T} B_{n}(\theta)
$$

We impose the following conditions:

Condition $1 \operatorname{Pr}\left[\sup _{\theta}\left|\frac{1}{T} B_{n}(\theta)\right| \geq \eta\right]=o\left(T^{-1}\right)$ for every $\eta>0$.

Condition $2 \sup _{\theta} \frac{1}{T}\left|\partial S_{n}(\theta) / \partial \theta^{\prime}\right|=o_{p}(1)$.

\section{Condition 3}

$$
\begin{aligned}
S_{n}\left(\theta_{0}\right)= & \frac{1}{n} \sum_{i=1}^{n} \sum_{l=-\infty}^{\infty} E\left[U_{i t}^{\gamma_{i}} \widetilde{V}_{i t-l}\right] \\
& +\frac{1}{2} \frac{1}{n} \sum_{i=1}^{n} E\left[U_{i}^{\gamma_{i} \gamma_{i}}\right] \operatorname{vec}\left(\sum_{l=-\infty}^{\infty} E\left[\widetilde{V}_{i t} \widetilde{V}_{i t-l}^{\prime}\right]\right)+o_{p}(1) .
\end{aligned}
$$

Under these conditions and the regularity conditions in Appendix A, we can obtain the asymptotic distribution of $\widetilde{\theta}$ as $n$ and $T$ grow at the same rate.

\footnotetext{
${ }^{5}$ This is a standard result, but we do provide a rigorous derivation in Supplementary Appendix, which is available upon request.
} 
Theorem 2 Assume that Conditions 1, 2, and 3 hold. Further assume that the regularity conditions in Appendix A hold. Finally, assume that $n / T \rightarrow \kappa$, where $0<\kappa<\infty$. Then

$$
\sqrt{n T}\left(\widetilde{\theta}-\theta_{0}\right) \Rightarrow N\left(0, \mathcal{I}^{-1} \Omega\left(\mathcal{I}^{\prime}\right)^{-1}\right)
$$

Proof. See Appendix B.

\subsection{Determinant Based Approach}

We now assume that $x_{i t}=\left(y_{i t}, y_{i, t-1}, \ldots, y_{i, t-q}\right)$ and $\psi\left(x_{i t} ; \theta, \gamma_{i}\right)=\ln p_{c}\left(y_{i t} \mid y_{i, t-1}, \ldots, y_{i, t-q} ; \theta, \gamma_{i}\right)$, where $p_{c}$ here denotes the conditional density of $y_{i t}$. We propose to estimate $\theta_{0}$ by

$$
\widetilde{\theta}=\underset{\theta}{\operatorname{argmax}} \sum_{i=1}^{n} \sum_{t=1}^{T} \psi\left(x_{i t} ; \theta, \widehat{\gamma}_{i}(\theta)\right)+\frac{1}{2} \sum_{i=1}^{n} \ln \operatorname{det} H_{i}\left(\theta, \widehat{\gamma}_{i}(\theta)\right)-\frac{1}{2} \sum_{i=1}^{n} \ln \operatorname{det} \Upsilon_{i}\left(\theta, \widehat{\gamma}_{i}(\theta)\right)
$$

where $H_{i}(\theta, \gamma)$ and $\Upsilon_{i}(\theta, \gamma)$ are as defined in (7)-(9).

Comparing (20) with (19), we obtain

$$
B_{n}(\theta)=-\frac{1}{2 n} \sum_{i=1}^{n} \ln \operatorname{det} H_{i}\left(\theta, \widehat{\gamma}_{i}(\theta)\right)+\frac{1}{2 n} \sum_{i=1}^{n} \ln \operatorname{det} \Upsilon_{i}\left(\theta, \widehat{\gamma}_{i}(\theta)\right)
$$

By differentiating $B_{n}$, we obtain $S_{n}\left(\theta_{0}\right)$. It can be shown that ${ }^{6}$

Theorem 3 Assume that the regularity conditions in Appendix A hold. Then, the $B_{n}(\theta)$ as defined in (21) satisfies Condition 1.

Theorem 4 Assume that the regularity conditions in Appendix A hold. Further assume that $m=$ $o\left(T^{1 / 2}\right)$. Then, the $B_{n}(\theta)$ as defined in (21) satisfies Condition 2.

Theorem 5 Assume that the model is given by the likelihood. Also assume that the regularity conditions in Appendix A hold. Further assume that $m=o\left(T^{2 / 5}\right)$. Then, the $B_{n}(\theta)$ as defined in (21) satisfies Condition 3.

Proof. See Appendix C.

Remark 1 Proof of Theorem 5 uses the information equality, as discussed in Appendix on page 18. This explains why the likelihood setup is required here.

Conclusion 1 Theorems 3, 4, and 5 imply that Theorem 2 applies to our new estimator.

\footnotetext{
${ }^{6}$ The proofs of Theorems 3 and 4 are in Supplementary Appendix, which is available upon request.
} 


\subsection{Expectation-based Determinant Approach}

We now consider a variant of the above estimator where instead of $\Upsilon_{i}(\theta, \gamma)$ we use

$$
\bar{\Upsilon}_{i}(\theta, \gamma) \equiv \sum_{l=-m}^{m} w_{T, l} E_{\widehat{\theta}, \widehat{\gamma}_{i}}\left[\frac{\partial \psi_{i t}(\theta, \gamma)}{\partial \gamma} \frac{\partial \psi_{i t-l}(\theta, \gamma)}{\partial \gamma^{\prime}}\right]
$$

Here, $E_{\widehat{\theta}, \widehat{\gamma}_{i}}[\cdot]$ denotes an expectation taken with respect to the density evaluated at $\left(\widehat{\theta}, \widehat{\gamma}_{i}\right)$. Note that $B_{n}(\theta)$ is defined similarly as in (21). As before, $B_{n}$, we obtain $S_{n}\left(\theta_{0}\right)$ by differentiating $B_{n}$. It can be shown that ${ }^{7}$

Theorem 6 Assume that the regularity conditions in Appendix A hold. Then the $B_{n}(\theta)$ based on (22) satisfies Condition 1 as long as $m \rightarrow \infty$ such that $m=o\left(T^{2 / 5}\right)$. The same result hold even when the preliminary estimates $\left(\widehat{\theta}, \widehat{\gamma}_{i}\right)$ in (22) are replaced by some $\left(\theta^{*}, \gamma_{i}^{*}\right)$ such that $\left\|\theta^{*}-\theta\right\|=O_{p}\left(T^{-2 / 5}\right)$ and $\sup _{i}\left\|\gamma_{i}^{*}-\gamma_{i 0}\right\|=O_{p}\left(T^{-2 / 5}\right)$.

Theorem 7 Assume that the regularity conditions in Appendix A hold. Further assume that $m=$ $o\left(T^{2 / 5}\right)$. Then, the $B_{n}(\theta)$ based on (22) satisfies Condition 2. The same result hold even when the preliminary estimates $\left(\widehat{\theta}, \widehat{\gamma}_{i}\right)$ in (22) are replaced by some $\left(\theta^{*}, \gamma_{i}^{*}\right)$ such that $\left\|\theta^{*}-\theta\right\|=O_{p}\left(T^{-2 / 5}\right)$ and $\sup _{i}\left\|\gamma_{i}^{*}-\gamma_{i 0}\right\|=O_{p}\left(T^{-2 / 5}\right)$.

Theorem 8 Assume that the model is given by the likelihood. Also assume that the regularity conditions in Appendix A hold. Further assume that $m=o\left(T^{2 / 5}\right)$. Then, the $B_{n}(\theta)$ as defined in (21) satisfies Condition 3. The same result hold even when the preliminary estimates $\left(\widehat{\theta}, \widehat{\gamma}_{i}\right)$ in (22) are replaced by some $\left(\theta^{*}, \gamma_{i}^{*}\right)$ such that $\left\|\theta^{*}-\theta\right\|=O_{p}\left(T^{-2 / 5}\right)$ and $\sup _{i}\left\|\gamma_{i}^{*}-\gamma_{i 0}\right\|=O_{p}\left(T^{-2 / 5}\right)$.

Proof. See Appendix G.

Remark 2 Proof of Theorem 5 uses the information equality, as discussed in Supplementary Appendix on page 22. This explains why we required the likelihood setup.

Conclusion 2 Theorems 6, 7, and 8 imply that Theorem 2 applies to our new estimator, even when the preliminary estimates $\left(\widehat{\theta}, \widehat{\gamma}_{i}\right)$ in (22) are replaced by some $\left(\theta^{*}, \gamma_{i}^{*}\right)$ satisfying some regularity condition.

\subsection{Trace Based Approach}

We now consider a slightly different approach where we set

$$
B_{n}(\theta)=\frac{1}{2 n} \sum_{i=1}^{n} \operatorname{trace}\left(H_{i}\left(\theta, \widehat{\gamma}_{i}(\theta)\right)^{-1} \Upsilon_{i}\left(\theta, \widehat{\gamma}_{i}(\theta)\right)\right)
$$

It can be shown that ${ }^{8}$

\footnotetext{
${ }^{7}$ The proof of Theorem 6 is in Supplementary Appendix, which is available upon request. The proof of Theorem 7 is similar to that of Theorem 4 , and is omitted.

${ }^{8}$ The proof of Theorem 9 is similar to those of Theorems 3 and 4 , and is omitted.
} 
Theorem 9 Assume that the regularity conditions in Appendix A hold. Further assume that $m=$ $o\left(T^{1 / 2}\right)$. Then, the $B_{n}(\theta)$ as defined in (23) satisfies Conditions 1 and 2.

Theorem 10 Assume that the regularity conditions in Appendix A hold. Further assume that $m=$ $o\left(T^{1 / 2}\right)$. Then, the $B_{n}(\theta)$ as defined in (23) satisfies Condition Condition 3.

Proof. See Appendix D.

Remark 3 Proof of Theorem 10 does not use the information equality. We therefore do not require the likelihood setup here.

\section{Concluding Remarks}

We discussed a modified objective function strategy to obtain estimators without bias to order $1 / T$ in nonlinear dynamic panel models with multiple effects. Estimation proceeds from a bias corrected objective function relative to some target infeasible criterion. We considered a determinant based approach for likelihood settings, and a trace based approach, which is not restricted to the likelihood setup. Both approaches depend exclusively on the Hessian and the outer product of the scores of the fixed effects. They produce simple and transparent corrections even in models with multiple effects.

We analyzed the asymptotic properties of the new estimators when $n$ and $T$ grow at the same rate, and showed that they are asymptotically normal and centered at the truth.

These approaches are likely to be useful in applications where the value of $T$ is not negligible relative to $n$, as is the case with many household, firm, and country-level panels. However, if $T / n$ is too small, further refinements may be required, because the sampling standard deviation of the $1 / T$ bias-corrected estimators will be small by comparison with the bias.

Existing Monte Carlo results and empirical estimates for binary choice and conditional volatility models are very encouraging, but more needs to be known about the properties of the new methods for other models and datasets. 


\section{Appendix}

\section{A Regularity Conditions}

Assumption 1 For each $\eta>0 \inf _{i}\left[G_{(i)}\left(\theta_{0}, \gamma_{i 0}\right)-\sup _{\left\{(\theta, \gamma):\left|(\theta, \gamma)-\left(\theta_{0}, \gamma_{i 0}\right)\right|>\eta\right\}} G_{(i)}(\theta, \gamma)\right]>0$.

Assumption $2 n, T \rightarrow \infty$ such that $\frac{n}{T} \rightarrow \kappa$, where $0<\kappa<\infty$.

Assumption 3 (i) For each $i,\left\{x_{i t}, t=1,2, \ldots\right\}$ is a stationary mixing sequence; (ii) $\left\{x_{i t}, t=1,2, \ldots\right\}$ are independent across i; (iii) $\sup _{i}\left|\alpha_{i}(m)\right| \leq C a^{m}$ for some a such that $0<a<1$ and some $C>0$, where $\mathcal{A}_{t}^{i} \equiv \sigma\left(x_{i t}, x_{i t-1}, x_{i t-2}, \ldots\right), \quad \mathcal{B}_{t}^{i} \equiv \sigma\left(x_{i t}, x_{i t+1}, x_{i t+2}, \ldots\right), \quad$ and $\alpha_{i}(m) \equiv \sup _{t} \sup _{A \in \mathcal{A}_{t}^{i}, B \in \mathcal{B}_{t+m}^{i}}|P(A \cap B)-P(A) P(B)|$.

Assumption 4 Let $\psi\left(x_{i t}, \phi\right)$ be a function indexed by the parameter $\phi=(\theta, \gamma) \in \operatorname{int} \Phi$, where $\Phi$ is a compact, convex subset of $\mathbb{R}^{p}, p \equiv \operatorname{dim}(\phi)$, and $R=\operatorname{dim}(\theta)$. Let $\nu=\left(\nu_{1}, \ldots, \nu_{k}\right)$ be a vector of nonnegative integers $v_{i},|v|=\sum_{j=1}^{k} v_{j}$ and $D^{v} \psi\left(x_{i t}, \phi\right)=\partial^{|\nu|} \psi\left(x_{i t}, \phi\right) /\left(\partial \phi_{1}^{v_{1}} \ldots \partial \phi_{k}^{\nu_{k}}\right)$. There exists a function $M\left(x_{i t}\right)$ such that $\left|D^{v} \psi\left(x_{i t}, \phi_{1}\right)-D^{v} \psi\left(x_{i t}, \phi_{2}\right)\right| \leq M\left(x_{i t}\right)\left\|\phi_{1}-\phi_{2}\right\|$ for all $\phi_{1}, \phi_{2} \in \Phi$ and $|v| \leq$ 5. The function $M\left(x_{i t}\right)$ satisfies $\sup _{\phi \in \Phi}\left\|D^{v} \psi\left(x_{i t}, \phi\right)\right\| \leq M\left(x_{i t}\right)$ and $\sup _{i} E\left[\left|M\left(x_{i t}\right)\right|^{10 q+12+\delta}\right]<\infty$ for some integer $q \geq p / 2+2$ and for some $\delta>0$.

Assumption 5 Let $\lambda_{i T}$ denote the smallest eigenvalue of $\Sigma_{i T}=\operatorname{Var}\left(T^{-1 / 2} \sum_{t=1}^{T} U_{i}\left(x_{i t} ; \theta, \gamma_{i}\right)\right)$. We assume that $\inf _{i} \inf _{T} \lambda_{i T}>0$.

Assumption 6 (i) $\inf _{i} \inf _{\theta, \gamma_{i}}\left|E\left[\partial^{2} \psi\left(x_{i t} ; \theta, \gamma_{i}\right) / \partial \gamma_{i} \partial \gamma_{i}^{\prime}\right]\right|>0$;

(ii) $\inf _{i} \inf _{\theta, \gamma_{i}} \sum_{l=-\infty}^{\infty} E\left[\left(\partial \psi\left(x_{i t} ; \theta, \gamma_{i}\right) / \partial \gamma_{i}\right)\left(\partial \psi\left(x_{i t-l} ; \theta, \gamma_{i}\right) / \partial \gamma_{i}^{\prime}\right)\right]>0$.

Remark 4 Assumption 6 is stronger than the one assumed in Hahn and Kuersteiner (2004).

Assumption 7 Let $\mu_{i 1} \leq \ldots \leq \mu_{i k} \leq \ldots \leq \mu_{i R}$ be the eigenvalues of $\mathcal{I}_{i}$ in ascending order. We have (i) $0<\inf _{i} \mu_{i 1} \leq \sup _{i} \mu_{i R}<\infty$; (ii) $\lim _{n \rightarrow \infty} n^{-1} \sum_{i=1}^{n} \mathcal{I}_{i}$ exists; (iii) letting $\mathcal{I} \equiv \lim _{n \rightarrow \infty} n^{-1} \sum_{i=1}^{n} \mathcal{I}_{i}$, we assume that $\mathcal{I}$ is positive definite.

Assumption $8 \sup _{(\theta, \gamma) \in \Phi} \sup _{l} E_{\theta, \gamma}\left[M\left(x_{i t}\right) M\left(x_{i t-l}\right)\right]<\infty$.

\section{B Proof of Theorem 2}

We focus on asymptotic normality here, taking consistency result as given. (The consistency result is available in a Supplementary Appendix, which is available upon request.) Because $0=$ $\sum_{t=1}^{T} V\left(x_{i t} ; \widetilde{\theta}, \widehat{\gamma}_{i}(\widetilde{\theta})\right)$ by definition, $\widetilde{\theta}$ can be given the alternative characterization

$$
0=\frac{1}{n T} \sum_{i=1}^{n} \sum_{t=1}^{T} U\left(x_{i t} ; \widetilde{\theta}, \widehat{\gamma}_{i}(\widetilde{\theta})\right)-\frac{1}{T} S_{n}(\widetilde{\theta}) .
$$


By the Taylor series expansion, we obtain

$$
\begin{aligned}
0= & \frac{1}{n T} \sum_{i=1}^{n} \sum_{t=1}^{T} U\left(x_{i t} ; \theta_{0}, \widehat{\gamma}_{i}\left(\theta_{0}\right)\right)-\frac{1}{T} S_{n}\left(\theta_{0}\right) \\
& +\left(\frac{1}{n T} \sum_{i=1}^{n} \sum_{t=1}^{T} U^{\theta}\left(x_{i t} ; \bar{\theta}, \widehat{\gamma}_{i}(\bar{\theta})\right)\right)\left(\widetilde{\theta}-\theta_{0}\right)-\frac{1}{T} \frac{\partial S_{n}(\bar{\theta})}{\partial \theta^{\prime}}\left(\widetilde{\theta}-\theta_{0}\right)
\end{aligned}
$$

for some $\bar{\theta}$ on the line segment adjoining $\theta_{0}$ and $\widetilde{\theta}$. Because $\mathcal{I}_{i} \equiv-E\left[\frac{\partial U_{i}\left(x_{i t} ; \theta_{0}, \gamma_{i 0}\right)}{\partial \theta^{\prime}}\right]$, we may define $\overline{\mathcal{I}}_{i} \equiv-\frac{1}{T} \sum_{t=1}^{T} U^{\theta}\left(x_{i t} ; \bar{\theta}, \widehat{\gamma}_{i}(\bar{\theta})\right)$, which yields

$$
\sqrt{n T}\left(\widetilde{\theta}-\theta_{0}\right)=\left(\frac{1}{n} \sum_{i=1}^{n} \overline{\mathcal{I}}_{i}+\frac{1}{T} \frac{\partial S_{n}(\bar{\theta})}{\partial \theta^{\prime}}\right)^{-1}\left(\frac{1}{\sqrt{n T}} \sum_{i=1}^{n} \sum_{t=1}^{T} U\left(x_{i t} ; \theta_{0}, \widehat{\gamma}_{i}\left(\theta_{0}\right)\right)-\sqrt{\frac{n}{T}} S_{n}\left(\theta_{0}\right)\right)
$$

It can be shown ${ }^{9}$ that $\frac{1}{n} \sum_{i=1}^{n} \overline{\mathcal{I}}_{i}=\mathcal{I}+o_{p}(1)$. By Condition 2, we also have $\frac{1}{T} \partial S_{n}(\bar{\theta}) / \partial \theta^{\prime}=o_{p}(1)$. We therefore have

$$
\frac{1}{n} \sum_{i=1}^{n} \overline{\mathcal{I}}_{i}+\frac{1}{T} \frac{\partial S_{n}(\bar{\theta})}{\partial \theta^{\prime}}=\mathcal{I}+o_{p}(1)
$$

By applying a second order Taylor series approximation to $\frac{1}{n T} \sum_{i=1}^{n} \sum_{t=1}^{T} U\left(x_{i t} ; \theta_{0}, \widehat{\gamma}_{i}\left(\theta_{0}\right)\right)$ around $\gamma_{i 0}$, and noting that $\widehat{\gamma}_{i}\left(\theta_{0}\right)-\gamma_{i 0}=-\left(E\left[\frac{\partial V_{i}}{\partial \gamma_{i}^{\prime}}\right]\right)^{-1}\left(\frac{1}{T} \sum_{t=1}^{T} V_{i t}\right)+o_{p}\left(\frac{1}{\sqrt{T}}\right)=\frac{1}{T} \sum_{t=1}^{T} \widetilde{V}_{i t}+o_{p}\left(\frac{1}{\sqrt{T}}\right)$, we can anticipate that ${ }^{10}$

$$
\begin{aligned}
& \frac{1}{\sqrt{n T}} \sum_{i=1}^{n} \sum_{t=1}^{T} U\left(x_{i t} ; \theta_{0}, \widehat{\gamma}_{i}\left(\theta_{0}\right)\right) \\
= & \frac{1}{\sqrt{n T}} \sum_{i=1}^{n} \sum_{t=1}^{T} U_{i t}+\sqrt{\frac{n}{T}} \frac{1}{n} \sum_{i=1}^{n}\left(\frac{1}{\sqrt{T}} \sum_{t=1}^{T} U_{i t}^{\gamma_{i}}\right)\left(\frac{1}{\sqrt{T}} \sum_{t=1}^{T} \widetilde{V}_{i t}\right) \\
& +\sqrt{\frac{n}{T}} \frac{1}{2} \frac{1}{n} \sum_{i=1}^{n} E\left[U_{i}^{\gamma_{i} \gamma_{i}}\right]\left[\left(\frac{1}{\sqrt{T}} \sum_{t=1}^{T} \widetilde{V}_{i t}\right) \otimes\left(\frac{1}{\sqrt{T}} \sum_{t=1}^{T} \widetilde{V}_{i t}\right)\right]+o_{p}(1)
\end{aligned}
$$

It can be shown that by using the same argument as in Hahn and Kuersteiner (2004) that

$$
\begin{array}{r}
\frac{1}{2} \frac{1}{n} \sum_{i=1}^{n} E\left[U_{i}^{\gamma_{i} \gamma_{i}}\right]\left[\left(\frac{1}{\sqrt{T}} \sum_{t=1}^{T} \widetilde{V}_{i t}\right) \otimes\left(\frac{1}{\sqrt{T}} \sum_{t=1}^{T} \widetilde{V}_{i t}\right)\right]+\frac{1}{n} \sum_{i=1}^{n}\left(\frac{1}{\sqrt{T}} \sum_{t=1}^{T} U_{i t}^{\gamma_{i}}\right)\left(\frac{1}{\sqrt{T}} \sum_{t=1}^{T} \widetilde{V}_{i t}\right) \\
=\frac{1}{n} \sum_{i=1}^{n} \sum_{l=-\infty}^{\infty} E\left[U_{i}^{\gamma_{i}} \widetilde{V}_{i t-l}\right]+\frac{1}{2} \frac{1}{n} \sum_{i=1}^{n} E\left[U_{i}^{\gamma_{i} \gamma_{i}}\right] \operatorname{vec}\left(\sum_{l=-\infty}^{\infty} E\left[\widetilde{V}_{i t} \widetilde{V}_{i t-l}^{\prime}\right]\right)+o_{p}(1)
\end{array}
$$

which, when combining (24), (25), (26) and Condition 3, yields

$$
\sqrt{n T}\left(\widetilde{\theta}-\theta_{0}\right)=\mathcal{I}^{-1}\left(\frac{1}{\sqrt{n T}} \sum_{i=1}^{n} \sum_{t=1}^{T} U\left(x_{i t} ; \theta_{0}, \gamma_{i 0}\right)\right)+o_{p}(1)
$$

from which the conclusion follows.

\footnotetext{
${ }^{9}$ See Lemma 6 in Supplementary Appendix A.

${ }^{10}$ In Supplementary Appendix C, we provide a rigorous proof of the expansion (26).
} 


\section{Proof of Theorem 5}

By differentiating $B_{n}$, we obtain that $S_{n}(\theta)=[2]+\cdots+[5]$, where

$$
\begin{aligned}
& {[2] \equiv-} \frac{1}{2} \frac{1}{n} \sum_{i=1}^{n}\left(\frac{1}{T} \sum_{t=1}^{T} \frac{\partial^{3} \psi_{i t}}{\partial \theta\left(\partial \gamma^{\prime} \otimes \partial \gamma^{\prime}\right)}\right) \operatorname{vec}\left(\left(\frac{1}{T} \sum_{t=1}^{T} \frac{\partial^{2} \psi_{i t}}{\partial \gamma \partial \gamma^{\prime}}\right)^{-1}\right) \\
& {[3] \equiv-\frac{1}{2} \frac{1}{n} \sum_{i=1}^{n} \frac{\partial \hat{\gamma}_{i}^{\prime}(\theta)}{\partial \theta}\left(\frac{1}{T} \sum_{t=1}^{T} \frac{\partial^{3} \psi_{i t}}{\partial \gamma\left(\partial \gamma^{\prime} \otimes \partial \gamma^{\prime}\right)}\right) \operatorname{vec}\left(\left(\frac{1}{T} \sum_{t=1}^{T} \frac{\partial^{2} \psi_{i t}}{\partial \gamma \partial \gamma^{\prime}}\right)^{-1}\right) } \\
& {[4] \equiv } \\
& \cdot \frac{1}{2} \frac{1}{n} \sum_{i=1}^{n}\left[\frac{1}{T} \sum_{l=-m}^{m} w_{T, l}\left(\sum_{t=\max (1, l+1)}^{\min (T, T+l)} \frac{\partial}{\partial \theta}\left(\left(\frac{\partial \psi_{i t}}{\partial \gamma^{\prime}}\right) \otimes\left(\frac{\partial \psi_{i t-l}}{\partial \gamma^{\prime}}\right)\right)\right)\right] \\
& \cdot \operatorname{vec}\left(\left(\frac{1}{T} \sum_{l=-m}^{m} w_{T, l}\left(\sum_{t=\max (1, l+1)}^{\min (T, T+l)} \frac{\partial \psi_{i t}}{\partial \gamma} \frac{\partial \psi_{i t-l}}{\partial \gamma^{\prime}}\right)\right)^{-1}\right)
\end{aligned}
$$

and

$$
\begin{aligned}
{[5] \equiv } & \frac{1}{2} \frac{1}{n} \sum_{i=1}^{n} \frac{\partial \widehat{\gamma}_{i}^{\prime}(\theta)}{\partial \theta}\left[\frac{1}{T} \sum_{l=-m}^{m} w_{T, l}\left(\sum_{t=\max (1, l+1)}^{\min (T, T+l)} \frac{\partial}{\partial \gamma}\left(\left(\frac{\partial \psi_{i t}}{\partial \gamma^{\prime}}\right) \otimes\left(\frac{\partial \psi_{i t-l}}{\partial \gamma^{\prime}}\right)\right)\right)\right] \\
& \cdot \operatorname{vec}\left(\left(\frac{1}{T} \sum_{l=-m}^{m} w_{T, l}\left(\sum_{t=\max (1, l+1)}^{\min (T, T+l)} \frac{\partial \psi_{i t}}{\partial \gamma} \frac{\partial \psi_{i t-l}}{\partial \gamma^{\prime}}\right)\right)^{-1}\right)
\end{aligned}
$$

We will often use the first order condition for $\widehat{\gamma}_{i}(\theta)$, which implies that

$$
\frac{\partial \widehat{\gamma}_{i}^{\prime}(\theta)}{\partial \theta}=-\left(\sum_{t=1}^{T} \frac{\partial^{2} \psi_{i t}\left(\theta, \widehat{\gamma}_{i}(\theta)\right)}{\partial \theta \partial \gamma^{\prime}}\right)\left(\sum_{t=1}^{T} \frac{\partial^{2} \psi_{i t}\left(\theta, \widehat{\gamma}_{i}(\theta)\right)}{\partial \gamma \partial \gamma^{\prime}}\right)^{-1}
$$

In the discussion below, all the terms $[2], \cdots,[5]$ will be evaluated at $\theta_{0}$. We first take care of the expansion of [2] $+[3]$. Note first that, by definition of $U_{i t}\left(\theta, \gamma_{i}\right)$, we have $\frac{\partial^{3} \psi_{i t}(\theta, \gamma)}{\partial \theta\left(\partial \gamma^{\prime} \otimes \partial \gamma^{\prime}\right)}=U_{i t}^{\gamma \gamma}+\rho_{i} V_{i t}^{\gamma \gamma}$, where $V_{i t}^{\gamma \gamma}\left(\theta, \gamma_{i}\right)=\frac{\partial^{2} V_{i t}\left(\theta, \gamma_{i}\right)}{\partial \gamma^{\prime} \otimes \partial \gamma^{\prime}}$. It turns out that all the averages over $t$ on the RHS of [2] is uniformly consistent over $i{ }^{11}$ We therefore obtain

$$
[2]=-\frac{1}{2} \frac{1}{n} \sum_{i=1}^{n}\left(E\left[U_{i t}^{\gamma \gamma}\right]+\rho_{i} E\left[V_{i t}^{\gamma \gamma}\right]\right) \operatorname{vec}\left(\left(E\left[V_{i t}^{\gamma}\right]\right)^{-1}\right)+o_{p}(1)
$$

The uniform consistency over $i$ combined with (27) also implies that

$$
\max _{i}\left|\frac{\partial \widehat{\gamma}_{i}^{\prime}(\theta)}{\partial \theta}+\rho_{i}\right|=o_{p}(1)
$$

Using the uniform consistency and equation (29), we obtain

$$
[3]=\frac{1}{2} \frac{1}{n} \sum_{i=1}^{n} \rho_{i} E\left[V_{i t}^{\gamma \gamma}\right] \operatorname{vec}\left(\left(E\left[V_{i t}^{\gamma}\right]\right)^{-1}\right)+o_{p}(1)
$$

${ }^{11}$ See Lemma 6 in Supplementary Appendix. 
Combining (28) and (30), we obtain

$$
[2]+[3]=-\frac{1}{2} \frac{1}{n} \sum_{i=1}^{n} E\left[U_{i t}^{\gamma \gamma}\right] \operatorname{vec}\left(\left(E\left[V_{i t}^{\gamma}\right]\right)^{-1}\right)+o_{p}(1)
$$

We now take care of the expansion of $[4]+[5]$. Note that

$$
\begin{gathered}
\frac{\partial}{\partial \theta}\left(\left(\frac{\partial \psi_{i t}(\theta, \gamma)}{\partial \gamma^{\prime}}\right) \otimes\left(\frac{\partial \psi_{i t-l}(\theta, \gamma)}{\partial \gamma^{\prime}}\right)\right)=\left(\frac{\partial^{2} \psi_{i t}(\theta, \gamma)}{\partial \theta \partial \gamma^{\prime}}\right) \otimes\left(\frac{\partial \psi_{i t-l}(\theta, \gamma)}{\partial \gamma^{\prime}}\right) \\
+\left(\frac{\partial \psi_{i t}(\theta, \gamma)}{\partial \gamma^{\prime}}\right) \otimes\left(\frac{\partial^{2} \psi_{i t-l}(\theta, \gamma)}{\partial \theta \partial \gamma^{\prime}}\right)=\left(U_{i t}^{\gamma}+\rho_{i} V_{i t}^{\gamma}\right) \otimes V_{i t-l}^{\prime}+V_{i t}^{\prime} \otimes\left(U_{i t-l}^{\gamma}+\rho_{i} V_{i t-l}^{\gamma}\right)
\end{gathered}
$$

and

$$
\frac{\partial}{\partial \gamma}\left(\left(\frac{\partial \psi_{i t}(\theta, \gamma)}{\partial \gamma^{\prime}}\right) \otimes\left(\frac{\partial \psi_{i t}(\theta, \gamma)}{\partial \gamma^{\prime}}\right)\right)=V_{i t}^{\gamma} \otimes V_{i t-l}^{\prime}+V_{i t}^{\prime} \otimes V_{i t-l}^{\gamma}
$$

we can write

$$
\begin{aligned}
{[4]+[5]=} & \frac{1}{2} \frac{1}{n} \sum_{i=1}^{n}\left[\frac{1}{T} \sum_{l=-m}^{m} w_{T, l} \sum_{t=\max (1, l+1)}^{\min (T, T+l)}\left(\begin{array}{c}
U_{i t}^{\gamma}\left(\theta_{0}, \widehat{\gamma}_{i}\left(\theta_{0}\right)\right) \otimes V_{i t-l}\left(\theta_{0}, \widehat{\gamma}_{i}\left(\theta_{0}\right)\right)^{\prime} \\
+V_{i t}\left(\theta_{0}, \widehat{\gamma}_{i}\left(\theta_{0}\right)\right)^{\prime} \otimes U_{i t-l}^{\gamma}\left(\theta_{0}, \widehat{\gamma}_{i}\left(\theta_{0}\right)\right)
\end{array}\right)\right] \\
& \cdot \operatorname{vec}\left(\left(\frac{1}{T} \sum_{l=-m}^{m} w_{T, l} \sum_{t=\max (1, l+1)}^{\min (T, T+l)} V_{i t}\left(\theta_{0}, \widehat{\gamma}_{i}\left(\theta_{0}\right)\right) V_{i t-l}\left(\theta_{0}, \widehat{\gamma}_{i}\left(\theta_{0}\right)\right)^{\prime}\right)^{-1}\right)+o_{p}(1)
\end{aligned}
$$

Using Lemma 5 in Supplementary Appendix, we obtain

$$
\max _{i}\left|\frac{1}{T} \sum_{l=-m}^{m} w_{T, l} \sum_{t=\max (1, l+1)}^{\min (T, T+l)} V_{i t}\left(\theta_{0}, \widehat{\gamma}_{i}\left(\theta_{0}\right)\right) V_{i t-l}\left(\theta_{0}, \widehat{\gamma}_{i}\left(\theta_{0}\right)\right)^{\prime}-\sum_{l=-\infty}^{\infty} E\left[V_{i t} V_{i t-l}^{\prime}\right]\right|=o_{p}(1)
$$

Furthermore, if the conditional likelihood is properly defined, then we should have $V_{i t}$ serially uncorrelated, which implies that

$$
\begin{aligned}
& \max _{i}\left|\frac{1}{T} \sum_{l=-m}^{m} w_{T, l} \sum_{t=\max (1, l+1)}^{\min (T, T+l)} V_{i t} V_{i t-l}^{\prime}-E\left[V_{i t} V_{i t}^{\prime}\right]\right| \\
&=\max _{i}\left|\frac{1}{T} \sum_{l=-m}^{m} w_{T, l} \sum_{t=\max (1, l+1)}^{\min (T, T+l)} V_{i t} V_{i t-l}^{\prime}+E\left[V_{i t}^{\gamma}\right]\right|=o_{p}(1)
\end{aligned}
$$

where the first equality is based on the information equality. Therefore, we obtain

$$
\begin{aligned}
{[4]+[5] } & =-\frac{1}{2} \frac{1}{n} \sum_{i=1}^{n}\left[\frac{1}{T} \sum_{l=-m}^{m} w_{T, l} \sum_{t=\max (1, l+1)}^{\min (T, T+l)}\left(\begin{array}{c}
U_{i t}^{\gamma}\left(\theta_{0}, \widehat{\gamma}_{i}\left(\theta_{0}\right)\right) \otimes V_{i t-l}\left(\theta_{0}, \widehat{\gamma}_{i}\left(\theta_{0}\right)\right)^{\prime} \\
+V_{i t}\left(\theta_{0}, \widehat{\gamma}_{i}\left(\theta_{0}\right)\right)^{\prime} \otimes U_{i t-l}^{\gamma}\left(\theta_{0}, \widehat{\gamma}_{i}\left(\theta_{0}\right)\right)
\end{array}\right)\right] \\
& \cdot \operatorname{vec}\left(E\left[V_{i t}^{\gamma}\right]^{-1}\right)+o_{p}(1)
\end{aligned}
$$


Using Lemma 5 again, we obtain

$$
\begin{aligned}
{[4]+[5] } & =-\frac{1}{2} \frac{1}{n} \sum_{i=1}^{n} \sum_{l=-\infty}^{\infty} E\left[U_{i t}^{\gamma} \otimes V_{i t-l}^{\prime}+V_{i t}^{\prime} \otimes U_{i t-l}^{\gamma}\right] \operatorname{vec}\left(E\left[V_{i t}^{\gamma}\right]^{-1}\right)+o_{p}(1) \\
& =\frac{1}{2} \frac{1}{n} \sum_{i=1}^{n} \sum_{l=-\infty}^{\infty} E\left[U_{i t}^{\gamma} \widetilde{V}_{i t-l}+U_{i t-l}^{\gamma} \widetilde{V}_{i t}\right]+o_{p}(1) \\
& =\frac{1}{n} \sum_{i=1}^{n} \sum_{l=-\infty}^{\infty} E\left[U_{i t}^{\gamma} \widetilde{V}_{i t-l}\right]+o_{p}(1)
\end{aligned}
$$

Combining (31) and (32), we obtain

$$
S_{n}\left(\theta_{0}\right)=-\frac{1}{2} \frac{1}{n} \sum_{i=1}^{n} E\left[U_{i t}^{\gamma \gamma}\right] \operatorname{vec}\left(\left(E\left[V_{i t}^{\gamma}\right]\right)^{-1}\right)+\frac{1}{n} \sum_{i=1}^{n} \sum_{l=-\infty}^{\infty} E\left[U_{i t}^{\gamma} \widetilde{V}_{i t-l}\right]+o_{p}(1)
$$

Now, we note that, under correct specification of conditional likelihood, the $\widetilde{V}_{i t}$ would have zero serial correlation and we would therefore have $\sum_{l=-\infty}^{\infty} E\left[\widetilde{V}_{i t} \widetilde{V}_{i t-l}^{\prime}\right]=E\left[\widetilde{V}_{i t} \widetilde{V}_{i t}^{\prime}\right]=\left(E\left[V_{i}^{\gamma}\right]\right)^{-1} E\left[V_{i t} V_{i t}^{\prime}\right]\left(E\left[V_{i}^{\gamma}\right]\right)^{-1}$. Furthermore, we have $E\left[V_{i t} V_{i t}^{\prime}\right]=-E\left[V_{i}^{\gamma}\right]$ by the information equality. It follows that

$$
S_{n}\left(\theta_{0}\right)=\frac{1}{2} \frac{1}{n} \sum_{i=1}^{n} E\left[U_{i}^{\gamma_{i} \gamma_{i}}\right] \operatorname{vec}\left(\sum_{l=-\infty}^{\infty} E\left[\widetilde{V}_{i t} \widetilde{V}_{i t-l}^{\prime}\right]\right)+\frac{1}{n} \sum_{i=1}^{n} \sum_{l=-\infty}^{\infty} E\left[U_{i t}^{\gamma} \widetilde{V}_{i t-l}\right]+o_{p}(1)
$$

\section{Proof of Theorem 10}

We have

$$
\begin{aligned}
S_{n}(\theta)= & \frac{1}{2 n} \sum_{i=1}^{n}\left(\frac{1}{T} \sum_{t=1}^{T} \frac{\partial^{3} \psi_{i t}}{\partial \theta\left(\partial \gamma^{\prime} \otimes \partial \gamma^{\prime}\right)}\right) \operatorname{vec}\left(H_{i}^{-1} \Upsilon_{i} H_{i}^{-1}\right) \\
& +\frac{1}{2 n} \sum_{i=1}^{n} \frac{\partial \widehat{\gamma}_{i}^{\prime}(\theta)}{\partial \theta}\left(\frac{1}{T} \sum_{t=1}^{T} \frac{\partial^{3} \psi_{i t}}{\partial \gamma\left(\partial \gamma^{\prime} \otimes \partial \gamma^{\prime}\right)}\right) \operatorname{vec}\left(H_{i}^{-1} \Upsilon_{i} H_{i}^{-1}\right) \\
& +\frac{1}{2 n} \sum_{i=1}^{n}\left(\frac{1}{T} \sum_{l=-m}^{m} \sum_{t=\max (1, l+1)}^{\min (T, T+l)} \frac{\partial}{\partial \theta}\left(\left(\frac{\partial \psi_{i t}}{\partial \gamma^{\prime}}\right) \otimes\left(\frac{\partial \psi_{i t-l}}{\partial \gamma^{\prime}}\right)\right)\right) \operatorname{vec}\left(H_{i}^{-1}\right) \\
& +\frac{1}{2 n} \sum_{i=1}^{n} \frac{\partial \widehat{\gamma}_{i}^{\prime}(\theta)}{\partial \theta}\left(\frac{1}{T} \sum_{l=-m}^{m} \sum_{t=\max (1, l+1)}^{\min (T, T+l)} \frac{\partial}{\partial \gamma}\left(\left(\frac{\partial \psi_{i t}}{\partial \gamma^{\prime}}\right) \otimes\left(\frac{\partial \psi_{i t-l}}{\partial \gamma^{\prime}}\right)\right)\right) \operatorname{vec}\left(H_{i}^{-1}\right)
\end{aligned}
$$

Proceeding as in Section C, we can obtain that

$$
\begin{aligned}
S_{n}\left(\theta_{0}\right)= & \frac{1}{2 n} \sum_{i=1}^{n} E\left[U_{i t}^{\gamma \gamma}\right] \operatorname{vec}\left(\left(E\left[V_{i t}^{\gamma}\right]\right)^{-1}\left(\sum_{l=-\infty}^{\infty} E\left[V_{i t} V_{i t-l}^{\prime}\right]\right)\left(E\left[V_{i t}^{\gamma}\right]\right)^{-1}\right) \\
& -\frac{1}{2 n} \sum_{i=1}^{n} \sum_{l=-\infty}^{\infty} E\left[U_{i t}^{\gamma} \otimes V_{i t-l}^{\prime}+V_{i t}^{\prime} \otimes U_{i t-l}^{\gamma}\right] \operatorname{vec}\left(\left(E\left[V_{i t}^{\gamma}\right]\right)^{-1}\right)+o_{p}(1) \\
= & \frac{1}{2 n} \sum_{i=1}^{n} E\left[U_{i t}^{\gamma \gamma}\right] \operatorname{vec}\left(\sum_{l=-\infty}^{\infty} E\left[\widetilde{V}_{i t} \widetilde{V}_{i t-l}^{\prime}\right]\right)+\frac{1}{2 n} \sum_{i=1}^{n} \sum_{l=-\infty}^{\infty} E\left[U_{i t}^{\gamma} \widetilde{V}_{i t-l}+U_{i t-l}^{\gamma} \widetilde{V}_{i t}\right]+o_{p}(1) \\
= & \frac{1}{2 n} \sum_{i=1}^{n} E\left[U_{i t}^{\gamma \gamma}\right] \operatorname{vec}\left(\sum_{l=-\infty}^{\infty} E\left[\widetilde{V}_{i t} \widetilde{V}_{i t-l}^{\prime}\right]\right)+\frac{1}{n} \sum_{i=1}^{n} \sum_{l=-\infty}^{\infty} E\left[U_{i t}^{\gamma} \widetilde{V}_{i t-l}\right]+o_{p}(1)
\end{aligned}
$$




\section{References}

[1] Arellano, M. (2003): "Discrete Choices with Panel Data", Investigaciones Económicas, 27, $423-458$.

[2] Arellano, M. and J. Hahn (2006): "Understanding Bias in Nonlinear Panel Models: Some Recent Developments". In: R. Blundell, W. Newey, and T. Persson (eds.): Advances in Economics and Econometrics, Ninth World Congress, Cambridge University Press, forthcoming.

[3] Bester, C. A. And C. Hansen (2005): "A Penalty Function Approach to Bias Reduction in Non-linear Panel Models with Fixed Effects", unpublished manuscript.

[4] Card, D. And D. R. Hyslop (2005): "Estimating the Effects of a Time-Limited Earnings Subsidy for Welfare-Leavers", Econometrica, 73, 1723-1770.

[5] Carro, J. (2006): "Estimating Dynamic Panel Data Discrete Choice Models with Fixed Effects", Journal of Econometrics, forthcoming.

[6] Chamberlain, G. (1985): "Heterogeneity, Omitted Variable Bias, and Duration Dependence", in J. J. Heckman and B. Singer (eds.), Longitudinal Analysis of Labor Market Data, Cambridge University Press.

[7] Cox, D. R. And N. ReID (1987): "Parameter Orthogonality and Approximate Conditional Inference" (with discussion), Journal of the Royal Statistical Society, Series B, 49, 1-39.

[8] DiCiccio, T. J. and S. E. Stern (1993): "An Adjustment to Profile Likelihood Based on Observed Information", Technical Report, Department of Statistics, Stanford University.

[9] DiCiccio, T. J., M. A. Martin, S. E. Stern, and G. A. Young (1996): "Information Bias and Adjusted Profile Likelihoods", Journal of the Royal Statistical Society, Series B, 58, 189-203.

[10] Ferguson, H., N. Reid, And D. R. Cox (1991): "Estimating Equations from Modified Profile Likelihood", in Godambe, V. P. (ed.), Estimating Functions, Oxford University Press.

[11] Fernández-Val, I. (2005): "Estimation of Structural Parameters and Marginal Effects in Binary Choice Panel Data Models with Fixed Effects", unpublished manuscript.

[12] Hahn, J., And G. Kuersteiner (2004): "Bias Reduction for Dynamic Nonlinear Panel Models with Fixed Effects", unpublished manuscript.

[13] Hahn, J. And W.K. Newey (2004): "Jackknife and Analytical Bias Reduction for Nonlinear Panel Models", Econometrica, 72, 1295-1319. 
[14] Hospido, L. (2006): "Modelling Heterogeneity and Dynamics in the Volatility of Individual Wages", unpublished manuscript.

[15] Neyman, J. and E. L. Scott (1948): "Consistent Estimates Based on Partially Consistent Observations", Econometrica, 16, 1-32.

[16] Pace, L. And A. Salvan (2005): "Adjustments of the Profile Likelihood from a New Perspective", Journal of Statistical Planning and Inference, forthcoming.

[17] Woutersen, T. (2002): "Robustness against Incidental Parameters", unpublished manuscript. 
Supplementary Appendix to "A likelihood-based approximate solution to the incidental parameter problem in dynamic nonlinear models with multiple effects"

Manuel Arellano and Jinyong Hahn 


\section{A Some Auxiliary Lemmas}

Throughout this appendix, we will let $F \equiv\left(F_{1}, \ldots, F_{n}\right)$ denote the collection of (marginal) distribution functions of $x_{i t}$ and $\widehat{F} \equiv\left(\widehat{F}_{1}, \ldots, \widehat{F}_{n}\right)$, where $\widehat{F}_{i}$ denotes the empirical distribution function for the $i$-th observation. Define $F(\epsilon) \equiv F+\epsilon \sqrt{T}(\widehat{F}-F)$ for $\epsilon \in\left[0, T^{-1 / 2}\right]$, and $\Delta_{i T} \equiv \sqrt{T}\left(\widehat{F}_{i}-F_{i}\right)$. We first provide a different version of Lahiri's (1992) Lemma 5.1, which is stated for bounded zero mean random variables.

Lemma 1 (Hahn and Kuersteiner, 2004) Assume that $\left\{W_{t}, t=1,2, \ldots\right\}$ is a stationary, mixing sequence with $E\left[W_{t}\right]=0$ and $E\left[\left|W_{t}\right|^{2 r+\delta}\right]<\infty$ for any positive integer $r$, some $\delta>0$ and all $t$. Let $\mathcal{A}_{t}=\sigma\left(W_{t}, W_{t-1}, W_{t-2}, \ldots\right), \mathcal{B}_{t}=\sigma\left(W_{t}, W_{t+1}, W_{t+2}, \ldots\right)$, and $\alpha(m)=\sup _{t} \sup _{A \in \mathcal{A}_{t}, B \in \mathcal{B}_{t+m}}|P(A \cap B)-P(A) P(B)|$. Then, for any $m$ such that $1 \leq m<C(r) n$,

$$
E\left[\left(\sum_{i=1}^{n} W_{i}\right)^{2 r}\right] \leq C(r) E\left[\left|W_{i}\right|^{2 r+\delta}\right]\left[n^{r} m^{2 r}+n^{2 r} \alpha(m)^{\frac{\delta}{2 r+\delta}}\right]
$$

where $C(r)$ is a constant that depends on $r$.

Lemma 2 (Hahn and Kuersteiner, 2004) Suppose that, for each $i,\left\{\xi_{i t}, t=1,2, \ldots\right\}$ is a mixing sequence with $E\left[\xi_{i t}\right]=0$ for all $i, t$. Let $\mathcal{A}_{t}^{i}=\sigma\left(\xi_{i t}, \xi_{i t-1}, \xi_{i t-2}, \ldots\right), \mathcal{B}_{t}^{i}=\sigma\left(\xi_{i t}, \xi_{i t+1}, \xi_{i t+2}, \ldots\right)$, and $\alpha_{i}(m)=\sup _{t} \sup _{A \in \mathcal{A}_{t}^{i}, B \in \mathcal{B}_{t+k}^{i}}|P(A \cap B)-P(A) P(B)|$. Assume that $\sup _{i}\left|\alpha_{i}(m)\right| \leq C a^{m}$ for some a such that $0<a<1$ and some $0<C<\infty$. We assume that $\left\{\xi_{i t}, t=1,2,3, \ldots\right\}$ are independent across $i$. We also assume that $n=O(T)$. Finally, assume that $E\left[\left|\xi_{i t}\right|^{6+\delta}\right]<\infty$ for some $\delta>0$. We then have

$$
\operatorname{Pr}\left[\max _{1 \leq i \leq n}\left|\frac{1}{T} \sum_{t=1}^{T} \xi_{i t}\right|>\eta\right]=o\left(T^{-1}\right)
$$

for every $\eta>0$. Now assume that $E\left[\left|\xi_{i t}\right|^{10 q+12+\delta}\right]<\infty$ for some $\delta>0$ and some integer $q \geq 1$. Then,

$$
\operatorname{Pr}\left[\max _{1 \leq i \leq n}\left|\frac{1}{\sqrt{T}} \sum_{t=1}^{T} \xi_{i t}\right|>\eta T^{\frac{1}{10}-v}\right]=o\left(T^{-q}\right)
$$

for every $\eta>0$ and $0<v<(100 q+120)^{-1}$.

Lemma 3 (Hahn and Kuersteiner, 2004) Let $\xi\left(x_{i t}, \phi\right)$ be a function indexed by the parameter $\phi \in \Phi$ where $\Phi$ is a convex subset of $\mathbb{R}^{p}$ with $E\left[\xi\left(x_{i t}, \phi\right)\right]=0$ for all $i, t$ and $\phi \in \Phi$. Assume that there exists a function $\mathbf{M}\left(x_{i t}\right)$ such that $\left|\xi\left(x_{i t}, \phi_{1}\right)-\xi\left(x_{i t}, \phi_{2}\right)\right| \leq \mathbf{M}\left(x_{i t}\right)\left\|\phi_{1}-\phi_{2}\right\|$ for all $\phi_{1}, \phi_{2} \in \Phi$ and $\sup _{\phi}\left|\xi\left(x_{i t}, \phi\right)\right| \leq \mathbf{M}\left(x_{i t}\right)$. For each $i$, let $x_{i t}$ be a $\alpha$-mixing process with exponentially decaying mixing coefficients $\underline{\alpha}_{i}(m)$ satisfying $\sup _{i}\left|\underline{\alpha}_{i}(m)\right| \leq C a^{m}$ for some a such that $0<a<1$ and some $0<C<\infty$. Let $q$ denote a positive integer such that $q \geq \frac{p+4}{2}$, where $p=\operatorname{dim} \phi$. We also assume that $E\left[\left|\mathbf{M}\left(x_{i t}\right)\right|^{10 q+12+\delta}\right]<\infty$ for some $\delta>0$. Finally, assume that $n=O(T)$. We then have 
$\operatorname{Pr}\left[\max _{i}\left|\frac{1}{\sqrt{T}} \sum_{t=1}^{T} \xi\left(x_{i t}, \phi_{i}\right)\right|>T^{\frac{1}{10}-v}\right]=o\left(T^{-1}\right)$ for $0<v<(100 q+120)^{-1}$. Here, $\left\{\phi_{i}\right\}$ is an arbitrary nonstochastic sequence in $\Phi$.

Lemma 4 (Hahn and Kuersteiner, 2004) Assume that $x_{i t}$ satisfies Assumption 3, and let $\xi\left(x_{i t}, \phi\right)$ be a function indexed by the parameter $\phi \in \operatorname{int} \Phi$, where $\Phi$ is a convex subset of $\mathbb{R}^{p}$. For any sequence $\phi_{i} \in \operatorname{int} \Phi$, assume $E\left[\xi\left(x_{i t}, \phi_{i}\right)\right]=0$. Further assume that $\sup _{\phi}\left\|\xi\left(x_{i t}, \phi\right)\right\| \leq \mathbf{M}\left(x_{i t}\right)$ for some $\mathbf{M}\left(x_{i t}\right)$ such that $E\left[\mathbf{M}\left(x_{i t}\right)^{4}\right]<\infty$. Let $\Sigma_{n T}=\sum_{i=1}^{n} \Sigma_{i T}^{\xi \xi}$ with $\Sigma_{i T}^{\xi \xi}=\operatorname{Var}\left(\frac{1}{\sqrt{T}} \sum_{t=1}^{T} \xi\left(x_{i t}, \phi_{i}\right)\right)$. Denote the smallest eigenvalue of $\Sigma_{i T}^{\xi \xi}$ by $\lambda_{i T}^{\xi}$, and assume that $\inf _{i} \inf _{T} \lambda_{i T}^{\xi}>0$. Then,

$$
\frac{1}{\sqrt{n T}} \sum_{i=1}^{n} \sum_{t=1}^{T} \xi\left(x_{i t}, \phi_{i}\right) \Rightarrow N\left(0, f^{\xi \xi}\right), \quad \text { and } \quad \sup _{i}\left\|\Sigma_{i T}^{\xi \xi}-f_{i}^{\xi \xi}\right\| \rightarrow 0,
$$

where $f^{\xi \xi} \equiv \lim n^{-1} \sum_{i=1}^{n} f_{i}^{\xi \xi}$ and $f_{i}^{\xi \xi} \equiv \sum_{j=-\infty}^{\infty} E\left[\xi\left(x_{i t}, \phi_{i}\right) \xi\left(x_{i t-j}, \phi_{i}\right)^{\prime}\right]$.

Lemma 5 Let $k_{i t}=k\left(x_{i t} ; \theta, \gamma_{i}(\theta)\right)$ and $\widehat{k}_{i t}=k\left(x_{i t} ; \theta, \widehat{\gamma}_{i}(\theta)\right)$ where $x_{i t}$ satisfies Assumption $3, k$ satisfies Assumption 4 and $\widehat{\theta}, \widehat{\gamma}_{i}$ are defined in (1). Assume that $E\left[k_{i t}\right]=0$ for $i, t$. Let $f_{i}^{k k} \equiv$ $\sum_{l=-\infty}^{\infty} E\left[k_{i t} k_{i t-l}^{\prime}\right]$ and $f^{k k} \equiv \lim _{n \rightarrow \infty} n^{-1} \sum_{i=1}^{n} f_{i}^{k k}$. Then,

$$
\sup _{\theta}\left|\frac{1}{n} \sum_{i=1}^{n}\left(\frac{1}{T} \sum_{l=-m}^{m} w_{T, l} \sum_{t=\max (1, l)}^{\min (T, T+l)} \widehat{k}_{i t} \widehat{k}_{i t-l}^{\prime}\right)-f^{k k}\right|=o_{p}(1),
$$

where $m, T \rightarrow \infty$ such that $m=o\left(T^{2 / 5}\right)$.

Proof. The proof is almost identical to a similar result found in Hahn and Kuersteiner (2004). Let $r_{1}=\max (1, l)$ and $r_{2}=\min (T, T+l)$ and define $K_{i, m}=\frac{1}{T} \sum_{l=-m}^{m} w_{T, l} \sum_{t=r_{1}}^{r_{2}} k_{i t} k_{i t-l}^{\prime}$.

We first show that $\frac{1}{n} \sum_{i=1}^{n} K_{i, m}-f^{k k}=o_{p}(1)$. This follows if $\frac{1}{n} \sum_{i=1}^{n} E\left[K_{i, m}\right]-f^{k k}=o(1)$ and $\operatorname{Var}\left(\frac{1}{n} \sum_{i=1}^{n} K_{i, m}\right)=o(1)$. Since $f^{k k}-n^{-1} \sum_{i=1}^{n} f_{i}^{k k}=o(1)$ by definition, we first consider

$$
\begin{aligned}
& \left\|E\left[K_{i, m}\right]-f_{i}^{k k}\right\| \\
\leq & \sum_{l=-m}^{m}\left|\frac{r_{2}-r_{1}+1}{T} w_{T, l}-1\right|\left\|E\left[k_{i t} k_{i t-l}^{\prime}\right]\right\|+\sum_{|l|>m}\left\|E\left[k_{i t} k_{i t-l}^{\prime}\right]\right\| \\
= & \sum_{l=-m}^{m}\left|\frac{T-|l|}{T}-\frac{T-|l|}{T}\left(1-w_{T, l}\right)-1\right|\left\|E\left[k_{i t} k_{i t-l}^{\prime}\right]\right\|+\sum_{|l|>m}\left\|E\left[k_{i t} k_{i t-l}^{\prime}\right]\right\| \\
= & \sum_{l=-m}^{m}\left|\frac{1-|l|}{T}-\frac{T-|l|}{T} \frac{|l|}{m+1}\right|\left\|E\left[k_{i t} k_{i t-l}^{\prime}\right]\right\|+\sum_{|l|>m}\left\|E\left[k_{i t} k_{i t-l}^{\prime}\right]\right\| \\
\leq & \sum_{l=-m}^{m}\left(\frac{|l|}{T}+\frac{T-|l|}{T} \frac{|l|}{m+1}\right)\left\|E\left[k_{i t} k_{i t-l}^{\prime}\right]\right\|+\sum_{|l|>m}\left\|E\left[k_{i t} k_{i t-l}^{\prime}\right]\right\| \\
\leq & \sum_{l=-m}^{m}\left(\frac{1}{T}+\frac{1}{m}\right)|l|\left\|E\left[k_{i t} k_{i t-l}^{\prime}\right]\right\|+\sum_{|l|>m}\left\|E\left[k_{i t} k_{i t-l}^{\prime}\right]\right\| \\
\leq & \sum_{l=-m}^{m} c_{1}\left(\frac{1}{T}+\frac{1}{m}\right)|l|\left(a^{\frac{\delta}{2+\delta}}\right)^{|l|}+\left(a^{\frac{\delta}{2+\delta}}\right)^{m} c_{2} \sum_{l=1}\left(a^{\frac{\delta}{2+\delta}}\right)^{l} \rightarrow 0 \text { as } m, T \rightarrow \infty
\end{aligned}
$$


where the last inequality follows from Condition 3 and the fact that

$$
\left|E\left[k_{i t, j_{1}} k_{i t-l, j_{2}}\right]\right| \leq 8\left(E\left[\left|k_{i t, j_{1}}\right|^{2+\delta}\right]\right)^{\frac{1}{2+\delta}}\left(E\left[\left|k_{i t-l, j_{2}}\right|^{2+\delta}\right]\right)^{\frac{1}{2+\delta}}\left(a^{\frac{\delta}{2+\delta}}\right)^{|l|}
$$

for any two elements $k_{i t, j_{1}}$ and $k_{i t-l, j_{2}}$ of $k_{i t}$ and $k_{i t-l}$ for some $\delta>0$, which can be proved by Corollary A.2 of Hall and Heyde (1980). Since the bound on $\left\|E\left[K_{i, m}\right]-f_{i}^{k k}\right\|$ is uniform it therefore follows that $\frac{1}{n} \sum_{i=1}^{n} E\left[K_{i, m}\right]-f^{k k}=o(1)$.

Next we show that

$$
\left\|\operatorname{Var}\left(\frac{1}{n} \sum_{i=1}^{n} K_{i, m}\right)\right\| \leq \frac{1}{n^{2}} \sum_{i=1}^{n}\left\|\operatorname{Var}\left(K_{i, m}\right)\right\|=o(1) .
$$

To show this we may assume without loss of generality that $k_{i t}$ is scalar. The variance can then be evaluated as

$$
\begin{aligned}
& \operatorname{Var}\left(K_{i, m}\right) \\
= & \frac{1}{T^{2}} \sum_{l_{1}, l_{2}=-m}^{m} w_{T, l_{1}} w_{T, l_{2}} \sum_{t_{1}, t_{2}=r_{1}}^{r_{2}}\left(E\left[k_{i t_{1}} k_{i t-l_{1}} k_{i t_{2}} k_{i t_{2}-l_{2}}\right]-E\left[k_{i t_{1}} k_{i t-l_{1}}\right] E\left[k_{i t_{2}} k_{i t_{2}-l_{2}}\right]\right) \\
= & \frac{1}{T^{2}} \sum_{l_{1}, l_{2}=-m}^{m} w_{T, l_{1}} w_{T, l_{2}} \sum_{t_{1}, t_{2}=r_{1}}^{r_{2}}\left(E\left[k_{i t_{1}} k_{i t_{2}}\right] E\left[k_{i t-l_{1}} k_{i t_{2}-l_{2}}\right]+E\left[k_{i t_{1}} k_{i t_{2}-l_{2}}\right] E\left[k_{i t_{2}} k_{i t-l_{1}}\right]\right) \\
& +\frac{1}{T^{2}} \sum_{l_{1}, l_{2}=-m}^{m} w_{T, l_{1}} w_{T, l_{2}} \sum_{t_{1}, t_{2}=r_{1}}^{r_{2}} \operatorname{Cum}\left(k_{i t_{1}} k_{i t-l_{1}} k_{i t_{2}} k_{i t_{2}-l_{2}}\right) \\
= & O(1)
\end{aligned}
$$

such that $\operatorname{Var}\left(K_{i, m}\right)$ is uniformly bounded in $i$. It now follows that $\frac{1}{n} \sum_{i=1}^{n} K_{i, m}-f^{k k}=o_{p}(1)$ by Markov's inequality.

Next we turn to showing that

$$
\frac{1}{n} \sum_{i=1}^{n} \frac{1}{T} \sum_{l=-m}^{m} w_{T, l} \sum_{t=r_{1}}^{r_{2}}\left(\widehat{k}_{i t} \widehat{k}_{i t-l}^{\prime}-k_{i t} k_{i t-l}^{\prime}\right)=o_{p}(1) .
$$

We use the decomposition

$$
\begin{aligned}
& \frac{1}{T} \sum_{l=-m}^{m} w_{T, l} \sum_{t=r_{1}}^{r_{2}}\left(\widehat{k}_{i t} \widehat{k}_{i t-l}^{\prime}-k_{i t} k_{i t-l}^{\prime}\right) \\
= & \frac{1}{T} \sum_{l=-m}^{m} w_{T, l} \sum_{t=r_{1}}^{r_{2}}\left(\widehat{k}_{i t}-k_{i t}\right)\left(\widehat{k}_{i t-l}-k_{i t-l}\right)^{\prime} \\
& +\frac{1}{T} \sum_{l=-m}^{m} w_{T, l} \sum_{t=r_{1}}^{r_{2}} k_{i t}\left(\widehat{k}_{i t-l}-k_{i t-l}\right)^{\prime}+\frac{1}{T} \sum_{l=-m}^{m} w_{T, l} \sum_{t=r_{1}}^{r_{2}}\left(\widehat{k}_{i t}-k_{i t}\right) k_{i t-l}^{\prime}
\end{aligned}
$$

We first consider the term $\frac{1}{T} \sum_{l=-m}^{m} w_{T, l} \sum_{t=r_{1}}^{r_{2}}\left(\widehat{k}_{i t}-k_{i t}\right) k_{i s}^{\prime}$. Use a first order Taylor approximation to

$$
\widehat{k}_{i t}-k_{i t}=k_{i t}^{\theta}(\widehat{\theta}-\theta)+k_{i t}^{\gamma}\left(\widehat{\gamma}_{i}-\gamma_{i 0}\right)
$$

where $k_{i t}^{\theta}=\partial k\left(x_{i t} ; \tilde{\theta}, \tilde{\gamma}_{i}\right) / \partial \theta^{\prime}$ and $k_{i t}^{\gamma}=\partial k\left(x_{i t} ; \tilde{\theta}^{\prime}, \tilde{\gamma}_{i}^{\prime}\right) / \partial \gamma$ with $\tilde{\theta}, \tilde{\gamma}_{i}, \tilde{\theta}^{\prime}, \tilde{\gamma}_{i}^{\prime}$ such that $\left\|\tilde{\theta}-\theta_{0}\right\| \leq$ $\left\|\hat{\theta}-\theta_{0}\right\|,\left\|\tilde{\theta}^{\prime}-\theta_{0}\right\| \leq\left\|\hat{\theta}-\theta_{0}\right\|$, etc. by the multivariate version of the mean value theorem. Note 
that each row of $\partial k\left(x_{i t} ; \tilde{\theta}, \tilde{\gamma}_{i}\right) / \partial \theta^{\prime}$ needs to be evaluated at a different $\tilde{\theta}$ but in slight abuse of notation we do not make this explicit. Then

$$
\begin{aligned}
& \frac{1}{T} \sum_{l=-m}^{m} w_{T, l} \sum_{t=r_{1}}^{r_{2}} \operatorname{vec}\left[\left(\widehat{k}_{i t}-k_{i t}\right) k_{i t-1}^{\prime}\right] \\
= & \frac{1}{T} \sum_{l=-m}^{m} w_{T, l} \sum_{t=r_{1}}^{r_{2}}\left(k_{i t-l} \otimes k_{i t}^{\theta}\right)(\widehat{\theta}-\theta) \\
& +\frac{\left(\widehat{\gamma}_{i}-\gamma_{i 0}\right)}{T} \sum_{l=-m}^{m} w_{T, l} \sum_{t=r_{1}}^{r_{2}} \operatorname{vec}\left[k_{i t}^{\gamma} k_{i t-l}^{\prime}\right]
\end{aligned}
$$

and consider $\frac{1}{T} \sum_{l=-m}^{m} w_{T, l} \sum_{t=r_{1}}^{r_{2}}\left(k_{i t-l} \otimes k_{i t}^{\theta}\right)$. Without loss of generality assume that $\left(k_{i t-l} \otimes k_{i t}^{\theta}\right)$ is a scalar. Then by the Cauchy-Schwartz inequality

$$
\begin{aligned}
\left|\frac{1}{T} \sum_{t=r_{1}}^{r_{2}} k_{i t-l} k_{i t}^{\theta}\right| & \leq\left(\frac{1}{T} \sum_{t=1}^{T} k_{i t-l}^{2}\right)^{1 / 2}\left(\frac{1}{T} \sum_{t=1}^{T} \sup _{\theta, \gamma}\left(\partial k\left(x_{i t} ; \theta, \gamma\right) / \partial \theta^{\prime}\right)^{2}\right)^{1 / 2} \\
& \leq\left(\frac{1}{T} \sum_{t=1}^{T} M\left(x_{i t-l}\right)^{2}\right)^{1 / 2}\left(\frac{1}{T} \sum_{t=1}^{T} M\left(x_{i t}\right)^{2}\right)^{1 / 2}
\end{aligned}
$$

such that $E\left[\left|\frac{1}{T} \sum_{t=r_{1}}^{r_{2}} k_{i t-l} k_{i t}^{\theta}\right|\right] \leq\left(\frac{1}{T} \sum_{t=1}^{T} E\left[M\left(x_{i t-l}\right)^{2}\right]\right)^{1 / 2}\left(\frac{1}{T} \sum_{t=1}^{T} E\left[M\left(x_{i t}\right)^{2}\right]\right)^{1 / 2}=O(1)$ uniformly in $i$. It thus follows from the Markov inequality that

$$
\frac{1}{n} \sum_{i=1}^{n} \frac{1}{T} \sum_{l=-m}^{m} w_{T, l} \sum_{t=r_{1}}^{r_{2}}\left(k_{i t-l} \otimes k_{i t}^{\theta}\right)(\widehat{\theta}-\theta)=O_{p}(m / T) .
$$

We now turn to the second term in (34). Noting that

$$
T^{2 / 5} \max _{i}\left|\widehat{\gamma}_{i}-\gamma_{i 0}\right|=o_{p}(1)
$$

by Lemma (7), we obtain

$$
\begin{aligned}
& \left|\frac{1}{n} \sum_{i=1}^{n} \frac{\left(\widehat{\gamma}_{i}-\gamma_{i 0}\right)}{T} \sum_{l=-m}^{m} w_{T, l} \sum_{t=r_{1}}^{r_{2}} \operatorname{vec}\left[k_{i t}^{\gamma} k_{i t-l}^{\prime}\right]\right| \\
\leq & \frac{1}{T^{7 / 5}} T^{2 / 5} \max _{i}\left|\widehat{\gamma}_{i}-\gamma_{i 0}\right| \cdot \frac{1}{n} \sum_{i=1}^{n} \sum_{l=-m}^{m} w_{T, l} \sum_{t=r_{1}}^{r_{2}}\left\|\operatorname{vec}\left[k_{i t}^{\gamma} k_{i t-l}^{\prime}\right]\right\| \\
\leq & o_{p}\left(T^{-7 / 5}\right) \cdot \frac{1}{n} \sum_{i=1}^{n} \sum_{l=-m}^{m} w_{T, l} \sum_{t=r_{1}}^{r_{2}} \operatorname{vec}\left[M_{i t} M_{i t-l}^{\prime}\right] \\
= & o_{p}\left(T^{-7 / 5}\right) \sum_{l=-m}^{m}\left(1-\frac{|l|}{m+1}\right)(T-|l|) \\
= & o_{p}\left(T^{-7 / 5}\right) O(T m) \\
= & o_{p}\left(\frac{m}{T^{2 / 5}}\right)
\end{aligned}
$$


We now turn to

$$
\begin{aligned}
& \frac{1}{T} \sum_{l=-m}^{m} w_{T, l} \sum_{t=r_{1}}^{r_{2}} \operatorname{vec}\left(\widehat{k}_{i t}-k_{i t}\right)\left(\widehat{k}_{i t-l}-k_{i t-l}\right)^{\prime} \\
= & \frac{1}{T} \sum_{l=-m}^{m} w_{T, l} \sum_{t=r_{1}}^{r_{2}}\left(k_{i t-l}^{\theta} \otimes k_{i t}^{\theta}\right) \operatorname{vec}(\widehat{\theta}-\theta)(\widehat{\theta}-\theta)^{\prime} \\
& +\frac{1}{T} \sum_{l=-m}^{m} w_{T, l} \sum_{t=r_{1}}^{r_{2}}\left(k_{i t-l}^{\theta} \otimes k_{i t}^{\gamma}\right)\left(\widehat{\gamma}_{i}-\gamma_{i 0}\right) \operatorname{vec}(\widehat{\theta}-\theta)^{\prime} \\
& +\frac{1}{T} \sum_{l=-m}^{m} w_{T, l} \sum_{t=r_{1}}^{r_{2}}\left(k_{i t-l}^{\gamma} \otimes k_{i t}^{\theta}\right)(\widehat{\theta}-\theta)\left(\widehat{\gamma}_{i}-\gamma_{i 0}\right) \\
& +\frac{1}{T} \sum_{l=-m}^{m} w_{T, l} \sum_{t=r_{1}}^{r_{2}}\left(\widehat{\gamma}_{i}-\gamma_{i 0}\right)^{2} \operatorname{vec}\left(k_{i t}^{\gamma} k_{i t-l}^{\gamma^{\prime}}\right)
\end{aligned}
$$

All the terms on the RHS are $o_{p}\left(m / T^{2 / 5}\right)$ by similar arguments.

Lemma 6 Under Assumptions 1, 2, 3, 4, 5, 6, and 7, we have

(i) $n^{-1} \sum_{i=1}^{n} \overline{\mathcal{I}}_{i}-\mathcal{I}=o_{p}(1)$;

(ii) $\max _{i}\left\|\frac{1}{T} \sum_{t=1}^{T} V_{i t}^{\gamma_{i}}\left(\theta_{0}, \widehat{\gamma}_{i}\left(\theta_{0}\right)\right)-E\left[V_{i}^{\gamma_{i}}\right]\right\|=o_{p}(1)$;

(iii) $\max _{i}\left\|\frac{1}{T} \sum_{t=1}^{T} V_{i t}^{\theta}\left(\theta_{0}, \widehat{\gamma}_{i}\left(\theta_{0}\right)\right)-E\left[V_{i}^{\theta}\right]\right\|=o_{p}(1)$;

(iv) $\max _{i}\left\|\frac{1}{T} \sum_{t=1}^{T} U_{i t}^{\gamma_{i} \gamma_{i}}\left(\theta_{0}, \widehat{\gamma}_{i}\left(\theta_{0}\right)\right)-E\left[U_{i}^{\gamma_{i} \gamma_{i}}\right]\right\|=o_{p}(1)$;

(v) $\max _{i}\left\|\frac{1}{T} \sum_{t=1}^{T} V_{i t}^{\gamma_{i} \gamma_{i}}\left(\theta_{0}, \widehat{\gamma}_{i}\left(\theta_{0}\right)\right)-E\left[V_{i}^{\gamma_{i} \gamma_{i}}\right]\right\|=o_{p}(1)$.

Proof. We only prove the first result. The rest can be proved using the same argument as in Hahn and Kuersteiner (2004). Note that

$$
\max _{i}\left\|\overline{\mathcal{I}}_{i}-\mathcal{I}_{i}\right\| \leq \sup _{i} E\left[\left\|M\left(x_{i t}\right)\right\|\right]\left(\|\bar{\theta}-\theta\|+\max _{i}\left|\widehat{\gamma}_{i}-\gamma_{i 0}\right|\right)+o_{p}(1) .
$$

Since

$$
\left|\widehat{\gamma}_{i}-\gamma_{i 0}\right| \leq \frac{1}{\sqrt{T}}\left|\widehat{\gamma}_{i}^{\epsilon}(0)\right|+\frac{1}{2 T}\left|\widehat{\gamma}_{i}^{\epsilon \epsilon}(\tilde{\epsilon})\right|
$$

with $\max _{i} T^{-\frac{1}{10}}\left|\widehat{\gamma}_{i}^{\epsilon}(0)\right|=o_{p}(1)$ and $\max _{i} T^{-\frac{2}{10}}\left|\widehat{\gamma}_{i}^{\epsilon \epsilon}(\tilde{\epsilon})\right|=o_{p}(1)$ by Lemma 14, it follows that $\max _{i}\left\|\overline{\mathcal{I}}_{i}-\mathcal{I}_{i}\right\|=$ $o_{p}(1)$ such that

$$
n^{-1} \sum_{i=1}^{n} \overline{\mathcal{I}}_{i}-\mathcal{I}=o_{p}(1) .
$$

Lemma 7 (Hahn and Kuersteiner, 2004) Let Assumptions 1, 2, 3, 4 and 5 be satisfied. Then $\operatorname{Pr}\left[\max _{i}\left|\sqrt{T}\left(\widehat{\gamma}_{i}-\gamma_{i 0}\right)\right|>T^{1 / 10-v}\right]=o\left(T^{-1}\right)$ for $0<v<(100 q+120)^{-1}$.

Lemma 8 Let Assumptions 1, 2, 3, 4 and 5 be satisfied. Then $\operatorname{Pr}\left[\max _{i}\left|\sqrt{T}\left(\widehat{\gamma}_{i}\left(\theta_{0}\right)-\gamma_{i 0}\right)\right|>T^{1 / 10-v}\right]=$ $o\left(T^{-1}\right)$ for $0<v<(100 q+120)^{-1}$. 
Proof. It can be proved in the same way as in Hahn and Kuersteiner (2004), and is omitted.

Lemma 9 Let $k_{i t}=k\left(x_{i t} ; \theta_{0}, \gamma_{i 0}\right)$ and $\widehat{k}_{i t}=k\left(x_{i t} ; \theta_{0}, \widehat{\gamma}_{i}\left(\theta_{0}\right)\right)$ where $x_{i t}$ satisfies Assumption $3, k_{i t}$ satisfies Assumption 4 and $\widehat{\theta}, \widehat{\gamma}_{i}$ are defined in (1). Assume that $E\left[k_{i t}\right]=0$ for $i, t$. Let $f_{i}^{k k}=$ $\sum_{l=-\infty}^{\infty} E\left[k_{i t} k_{i t-l}^{\prime}\right]$. Then, $\sup _{i}\left\|\sum_{l=-m}^{m} w_{T, l} E_{\widehat{\theta}, \widehat{\gamma}_{i}}\left[\widehat{k}_{i t} \widehat{k}_{i t-l}^{\prime}\right]-f^{k k}\right\|=o_{p}(1)$, where $m, T \rightarrow \infty$ such that $m=o\left(T^{2 / 5}\right)$.

Proof. For notational simplicity, we may assume without loss of generality that $k_{i t}$ is scalar. Let $K_{i, m}=\sum_{l=-m}^{m} w_{T, l} E\left[k_{i t} k_{i t-l}\right]$. We first consider

$$
\begin{aligned}
& \left\|K_{i, m}-f_{i}^{k k}\right\| \\
\leq & \sum_{l=-m}^{m}\left|\frac{r_{2}-r_{1}+1}{T} w_{T, l}-1\right|\left\|E\left[k_{i t} k_{i t-l}\right]\right\|+\sum_{|l|>m}\left\|E\left[k_{i t} k_{i t-l}\right]\right\| \\
\leq & \sum_{l=-m}^{m}\left(\frac{1}{T}+\frac{1}{m}\right)|l|\left\|E\left[k_{i t} k_{i t-l}\right]\right\|+\sum_{|l|>m}\left\|E\left[k_{i t} k_{i t-l}\right]\right\| \\
\leq & \sum_{l=-m}^{m} c_{1}\left(\frac{1}{T}+\frac{1}{m}\right)|l|\left(a^{\frac{\delta}{2+\delta}}\right)^{|l|}+\left(a^{\frac{\delta}{2+\delta}}\right)^{m} c_{2} \sum_{l=1}\left(a^{\frac{\delta}{2+\delta}}\right)^{l} \rightarrow 0 \text { as } m, T \rightarrow \infty
\end{aligned}
$$

where the last inequality follows from Assumption 3 and the fact that, for any two elements $k_{i t, j_{1}}$ and $k_{i t-l, j_{2}}$ of $k_{i t}$ and $k_{i t-l}$, it follows from Corollary A.2 of Hall and Heyde (1980) that

$$
\left|E\left[k_{i t, j_{1}} k_{i t-l, j_{2}}\right]\right| \leq 8\left(E\left[\left|k_{i t, j_{1}}\right|^{2+\delta}\right]\right)^{\frac{1}{2+\delta}}\left(E\left[\left|k_{i t-l, j_{2}}\right|^{2+\delta}\right]\right)^{\frac{1}{2+\delta}}\left(a^{\frac{\delta}{2+\delta}}\right)^{|l|}
$$

for some $\delta>0$. It follows that

$$
\sup _{i}\left\|K_{i, m}-f_{i}^{k k}\right\|=o(1) .
$$

Now, let

$$
\widehat{K}_{i, m}=\sum_{l=-m}^{m} w_{T, l} E_{\widehat{\theta}, \widehat{\gamma}_{i}}\left[\widehat{k}_{i t} \widehat{k}_{i t-l}\right]=\sum_{l=-m}^{m} w_{T, l} \int \widehat{k}_{i t} \widehat{k}_{i t-l} \widehat{p}_{i, t, l} d\left(x_{i t}, x_{i t-l}\right) .
$$

where

$$
\begin{aligned}
\widehat{k}_{i t} & \equiv k_{i t}\left(x_{i t} ; \theta_{0}, \widehat{\gamma}_{i}\left(\theta_{0}\right)\right) \\
\widehat{p}_{i, t, l} & \equiv p_{i, t, l}\left(x_{i t}, x_{i t-l} ; \widehat{\theta}, \widehat{\gamma}_{i}\right)
\end{aligned}
$$

Here, $p_{i, t, l}\left(x_{i t}, x_{i t-l} ; \theta, \gamma_{i}\right)$ denotes the joint density of $\left(x_{i t}, x_{i t-l}\right)$. Consider $\widehat{K}_{i, m}-K_{i, m}$

$$
\widehat{K}_{i, m}-K_{i, m}=\sum_{l=-m}^{m} w_{T, l} \int\left(\widehat{k}_{i t} \widehat{k}_{i t-l} \widehat{p}_{i t}-k_{i t} k_{i t-l} p_{i t}\right) d\left(x_{i t}, x_{i t-l}\right)
$$


We use the mean value theorem and write

$$
\begin{aligned}
\widehat{k}_{i t} \widehat{k}_{i t-l} \widehat{p}_{i t}-k_{i t} k_{i t-l} p_{i t}= & \widetilde{k}_{i t}^{\gamma} \widetilde{k}_{i t-l} \widetilde{p}_{i t}\left(\widehat{\gamma}_{i}\left(\theta_{0}\right)-\gamma_{i 0}\right)+\widetilde{k}_{i t} \widetilde{k}_{i t-l}^{\gamma} \widetilde{p}_{i t}\left(\widehat{\gamma}_{i}\left(\theta_{0}\right)-\gamma_{i 0}\right) \\
& +\widetilde{k}_{i t} \widetilde{k}_{i t-l} \widetilde{p}_{i t}^{\theta}(\widehat{\theta}-\theta)+\widetilde{k}_{i t} \widetilde{k}_{i t-l} \widetilde{p}_{i t}^{\gamma}\left(\widehat{\gamma}_{i}-\gamma_{i 0}\right)
\end{aligned}
$$

where $\widetilde{k}_{i t}^{\theta}=\partial k\left(x_{i t} ; \tilde{\theta}, \tilde{\gamma}_{i}\right) / \partial \theta$, etc. Note that we may write $\widetilde{p}_{i t}^{\theta}=\widetilde{u}_{i t}^{\theta} \widetilde{p}_{i t}$ and $\widetilde{f}_{i t}^{\gamma}=\widetilde{v}_{i t}^{\theta} \widetilde{p}_{i t}$. By Assumptions 4 and 8 , we obtain

$$
\left\|\widehat{K}_{i, m}-K_{i, m}\right\| \leq m \mathbf{M}\left(\sup _{i}\left\|\widehat{\gamma}_{i}\left(\theta_{0}\right)-\gamma_{i 0}\right\|+\|\widehat{\theta}-\theta\|+\sup _{i}\left\|\widehat{\gamma}_{i}-\gamma_{i 0}\right\|\right)
$$

for some finite constant $\mathbf{M}$, or

$$
\max _{i}\left\|\widehat{K}_{i, m}-K_{i, m}\right\|=O_{p}\left(\frac{m}{T^{2 / 5}}\right)
$$

by Lemmas 7 and 8 .

Lemma 10 Let $k_{i t}=k\left(x_{i t} ; \theta_{0}, \gamma_{i 0}\right)$ and $\widehat{k}_{i t}=k\left(x_{i t} ; \theta_{0}, \widehat{\gamma}_{i}\left(\theta_{0}\right)\right)$ where $x_{i t}$ satisfies Assumption 3, $k_{i t}$ satisfies Assumption 4 and $\theta^{*}, \gamma_{i}^{*}$ are such that $\left\|\theta^{*}-\theta\right\|=O_{p}\left(T^{-2 / 5}\right)$ and $\sup _{i}\left\|\gamma_{i}^{*}-\gamma_{i 0}\right\|=$ $O_{p}\left(T^{-2 / 5}\right)$. Then, $\sup _{i}\left\|\sum_{l=-m}^{m} w_{T, l} E_{\theta^{*}, \gamma_{i}^{*}}\left[\widehat{k}_{i t} \widehat{k}_{i t-l}^{\prime}\right]-f^{k k}\right\|=o_{p}(1)$, where $m, T \rightarrow \infty$ such that $m=o\left(T^{2 / 5}\right)$.

Proof. Similar to the proof of Lemma 9, and omitted.

Lemma 11 (Hahn and Kuersteiner, 2004) $\operatorname{Pr}\left[\max _{1 \leq i \leq n} \max _{0 \leq \epsilon \leq \frac{1}{\sqrt{T}}}\left|\widehat{\gamma}_{i}(\epsilon)-\gamma_{i 0}\right| \geq \eta\right]=o\left(T^{-1}\right)$ for every $\eta>0$.

Lemma 12 Suppose that $K_{i}\left(\cdot ; \theta_{0}, \gamma_{i}\left(\theta_{0}, \epsilon\right)\right)$ is equal to

$$
\frac{\partial^{m_{1}+m_{2}} \psi\left(x_{i t} ; \theta_{0}, \gamma_{i}\left(\theta_{0}, \epsilon\right)\right)}{\partial \gamma_{i}^{m}}
$$

for some $m \leq 1, \ldots, 5$. Then, for any $\eta>0$, we have

$$
\operatorname{Pr}\left[\max _{0 \leq \epsilon \leq \frac{1}{\sqrt{T}}}\left|\frac{1}{n} \sum_{i=1}^{n} \int K_{i}\left(\cdot ; \theta_{0}, \gamma_{i}\left(\theta_{0}, \epsilon\right)\right) d F_{i}(\epsilon)-\frac{1}{n} \sum_{i=1}^{n} E\left[K_{i}\left(x_{i t} ; \theta_{0}, \gamma_{i 0}\right)\right]\right|>\eta\right]=o\left(T^{-1}\right)
$$

and

$$
\operatorname{Pr}\left[\max _{i} \max _{0 \leq \epsilon \leq \frac{1}{\sqrt{T}}}\left|\int K_{i}\left(\cdot ; \theta_{0}, \gamma_{i}\left(\theta_{0}, \epsilon\right)\right) d F_{i}(\epsilon)-E\left[K_{i}\left(x_{i t} ; \theta_{0}, \gamma_{i 0}\right)\right]\right|>\eta\right]=o\left(T^{-1}\right) .
$$

Also,

$$
\operatorname{Pr}\left[\max _{i} \max _{0 \leq \epsilon \leq \frac{1}{\sqrt{T}}}\left|\int K_{i}\left(\cdot ; \theta_{0}, \gamma_{i}\left(\theta_{0}, \epsilon\right)\right) d \Delta_{i T}\right|>C T^{\frac{1}{10}-v}\right]=o\left(T^{-1}\right)
$$

for some constant $C>0$ and $0<v<(100 q+120)^{-1}$. 
Proof. Note that we may write

$$
\begin{aligned}
& \left\|\int K_{i}\left(\cdot ; \theta_{0}, \gamma_{i}\left(\theta_{0}, F_{i}(\epsilon)\right)\right) d F_{i}(\epsilon)-\int K_{i}\left(\cdot ; \theta_{0}, \gamma_{i 0}\right) d F_{i}\right\| \\
\leq & \left\|\int K_{i}\left(\cdot ; \theta_{0}, \gamma_{i}\left(\theta_{0}, F_{i}(\epsilon)\right)\right) d F_{i}(\epsilon)-\int K_{i}\left(\cdot ; \theta_{0}, \gamma_{i 0}\right) d F_{i}(\epsilon)\right\| \\
& +\left\|\int K_{i}\left(\cdot ; \theta_{0}, \gamma_{i 0}\right) d F_{i}(\epsilon)-\int K_{i}\left(\cdot ; \theta_{0}, \gamma_{i 0}\right) d F_{i}\right\| \\
\leq & \int M\left(x_{i t}\right)\left(\left|\gamma_{i}\left(\theta_{0}, F_{i}(\epsilon)\right)-\gamma_{i 0}\right|\right) d\left|F_{i}(\epsilon)\right| \\
& +\epsilon \sqrt{T}\left\|\int K_{i}\left(\cdot ; \theta_{0}, \gamma_{i 0}\right) d\left(\widehat{F}_{i}-F_{i}\right)\right\| .
\end{aligned}
$$

Therefore, we have

$$
\begin{aligned}
& \left\|\frac{1}{n} \sum_{i=1}^{n} \int K_{i}\left(\cdot ; \theta_{0}, \gamma_{i}\left(\theta_{0}, F_{i}(\epsilon)\right)\right) d F_{i}(\epsilon)-\int K_{i}\left(\cdot ; \theta_{0}, \gamma_{i 0}\right) d F_{i}\right\| \\
\leq & \left(\frac{1}{n} \sum_{i=1}^{n}\left(\gamma_{i}\left(\theta_{0}, F_{i}(\epsilon)\right)-\gamma_{i 0}\right)^{2}\right)^{1 / 2}\left(\frac{1}{n} \sum_{i=1}^{n}\left(E\left[M\left(x_{i t}\right)\right]+\frac{1}{T} \sum_{t=1}^{T} M\left(x_{i t}\right)\right)^{2}\right)^{1 / 2} \\
& +\left\|\frac{1}{n} \sum_{i=1}^{n}\left(\frac{1}{T} \sum_{t=1}^{T} K_{i}\left(x_{i t} ; \theta_{0}, \gamma_{i 0}\right)-E\left[K_{i}\left(x_{i t} ; \theta_{0}, \gamma_{i 0}\right)\right]\right)\right\|,
\end{aligned}
$$

the RHS of which can be bounded by using Lemmas 2 and 11 in absolute value by some $\eta>0$ with probability $1-o\left(T^{-1}\right)$.

Because

$$
\begin{aligned}
& \left|\int K_{i}\left(\cdot ; \theta_{0}, \gamma_{i}\left(\theta_{0}, F_{i}(\epsilon)\right)\right) d F_{i}(\epsilon)-E\left[K_{i}\left(x_{i t} ; \theta_{0}, \gamma_{i 0}\right)\right]\right| \\
\leq & \left|\gamma_{i}\left(\theta_{0}, F_{i}(\epsilon)\right)-\gamma_{i}\right| \cdot\left(E\left[M\left(x_{i t}\right)\right]+\frac{1}{T} \sum_{t=1}^{T} M\left(x_{i t}\right)\right) \\
& +\left|\frac{1}{T} \sum_{t=1}^{T} M\left(x_{i t}\right)-E\left[M\left(x_{i t}\right)\right]\right|
\end{aligned}
$$

we can bound

$$
\max _{i} \max _{0 \leq \epsilon \leq \frac{1}{\sqrt{T}}}\left|\int K_{i}\left(\cdot ; \theta_{0}, \gamma_{i}\left(\theta_{0}, F_{i}(\epsilon)\right)\right) d F_{i}(\epsilon)-E\left[K_{i}\left(x_{i t} ; \theta_{0}, \gamma_{i 0}\right)\right]\right|
$$

in absolute value by some $\eta>0$ with probability $1-o\left(T^{-1}\right)$.

Using Lemmas 3, we can also show that

$$
\max _{i}\left|\int K_{i}\left(\cdot ; \theta_{0}, \gamma_{i}\left(\theta_{0}, F_{i}(\epsilon)\right)\right) d \Delta_{i T}\right|
$$

can be bounded by in absolute value by $C T^{\frac{1}{10}-v}$ for some constant $C>0$ and $v$ such that $0 \leq v<\frac{1}{160}$ with probability $1-o\left(T^{-1}\right)$. 


\section{B Consistency}

Let

$$
\widehat{G}_{(i)}(\theta, \gamma) \equiv \frac{1}{T} \sum_{t=1}^{T} \psi\left(x_{i t} ; \theta, \gamma\right), \quad G_{(i)}(\theta, \gamma) \equiv E\left[\psi\left(x_{i t} ; \theta, \gamma\right)\right]
$$

where $\widehat{\gamma}_{i}(\theta) \equiv \operatorname{argmax}_{a} \sum_{t=1}^{T} \psi\left(x_{i t} ; \theta, a\right)$.

Lemma 13 (Hahn and Kuersteiner, 2004) For all $\eta>0$, it follows that

$$
\operatorname{Pr}\left[\max _{1 \leq i \leq n} \sup _{(\theta, \gamma)}\left|\widehat{G}_{(i)}(\theta, \gamma)-G_{(i)}(\theta, \gamma)\right| \geq \eta\right]=o\left(T^{-1}\right)
$$

Recall now that $\tilde{\theta}$ is a solution to (19).

Theorem $11 \operatorname{Pr}\left[\left|\widetilde{\theta}-\theta_{0}\right| \geq \eta\right]=o\left(T^{-1}\right)$ for every $\eta>0$.

Proof. Let $\eta$ be given, and let $\varepsilon \equiv \inf _{i}\left[G_{(i)}\left(\theta_{0}, \gamma_{i 0}\right)-\sup _{\left\{(\theta, \gamma):\left|(\theta, \gamma)-\left(\theta_{0}, \gamma_{i 0}\right)\right|>\eta\right\}} G_{(i)}(\theta, \gamma)\right]>0$. Because of Condition 1, we have

$$
\left|\frac{1}{T} B_{n}(\theta)\right| \leq \frac{1}{6} \varepsilon
$$

with probability equal to $1-o\left(\frac{1}{T}\right)$. Also, because of Lemma 13, we have

$$
\max _{1 \leq i \leq n} \sup _{(\theta, \gamma)}\left|\widehat{G}_{(i)}(\theta, \gamma)-G_{(i)}(\theta, \gamma)\right| \leq \frac{1}{6} \varepsilon
$$

with probability equal to $1-o\left(\frac{1}{T}\right)$. It follows that

$$
\begin{aligned}
& \max _{\left|\theta-\theta_{0}\right|>\eta, \gamma_{1}, \ldots, \gamma_{n}} n^{-1} \sum_{i=1}^{n} \widehat{G}_{(i)}\left(\theta, \gamma_{i}\right)-\frac{1}{T} B_{n}(\theta) \\
\leq & \max _{\left|\left(\theta, \gamma_{i}\right)-\left(\theta_{0}, \gamma_{i 0}\right)\right|>\eta} n^{-1} \sum_{i=1}^{n} \widehat{G}_{(i)}\left(\theta, \gamma_{i}\right)-\frac{1}{T} B_{n}(\theta) \\
\leq & \max _{\left|\left(\theta, \gamma_{i}\right)-\left(\theta_{0}, \gamma_{i 0}\right)\right|>\eta} n^{-1} \sum_{i=1}^{n} \widehat{G}_{(i)}\left(\theta, \gamma_{i}\right)+\frac{1}{6} \varepsilon \\
\leq & \max _{\left|\left(\theta, \gamma_{i}\right)-\left(\theta_{0}, \gamma_{i 0}\right)\right|>\eta} n^{-1} \sum_{i=1}^{n} G_{(i)}\left(\theta, \gamma_{i}\right)+\frac{1}{3} \varepsilon \\
\leq & n^{-1} \sum_{i=1}^{n} G_{(i)}\left(\theta_{0}, \gamma_{i 0}\right)-\frac{2}{3} \varepsilon \\
\leq & n^{-1} \sum_{i=1}^{n} \widehat{G}_{(i)}\left(\theta_{0}, \gamma_{i 0}\right)-\frac{1}{T} B_{n}\left(\theta_{0}\right)-\frac{1}{3} \varepsilon
\end{aligned}
$$


Because

$$
\max _{\theta, \gamma_{1}, \ldots, \gamma_{n}} n^{-1} \sum_{i=1}^{n} \widehat{G}_{(i)}\left(\theta, \gamma_{i}\right)-\frac{1}{T} B_{n}(\theta) \geq n^{-1} \sum_{i=1}^{n} \widehat{G}_{(i)}\left(\theta_{0}, \gamma_{i 0}\right)-\frac{1}{T} B_{n}\left(\theta_{0}\right)
$$

by definition, we can conclude that $\operatorname{Pr}\left[\left|\widetilde{\theta}-\theta_{0}\right| \geq \eta\right]=o\left(T^{-1}\right)$.

Theorem 12 (Hahn and Kuersteiner, 2004) $\operatorname{Pr}\left[\max _{1 \leq i \leq n}\left|\widehat{\gamma}_{i}-\gamma_{i 0}\right| \geq \eta\right]=o\left(T^{-1}\right)$

Theorem 13 Let $\bar{\theta}$ be such that $\operatorname{Pr}\left[\left|\bar{\theta}-\theta_{0}\right| \geq \eta\right]=o\left(T^{-1}\right)$ for every $\eta>0$. Then,

$$
\operatorname{Pr}\left[\max _{1 \leq i \leq n}\left|\widehat{\gamma}_{i}(\bar{\theta})-\gamma_{i 0}\right| \geq \eta\right]=o\left(T^{-1}\right)
$$

for every $\eta>0$.

Proof. We first prove that

$$
T \operatorname{Pr}\left[\max _{1 \leq i \leq n} \sup _{\gamma}\left|\widehat{G}_{(i)}(\bar{\theta}, \gamma)-G_{(i)}\left(\theta_{0}, \gamma\right)\right| \geq \eta\right]=o(1)
$$

for every $\eta>0$. Note that

$$
\begin{aligned}
& \max _{1 \leq i \leq n} \sup _{\gamma}\left|\widehat{G}_{(i)}(\bar{\theta}, \gamma)-G_{(i)}\left(\theta_{0}, \gamma\right)\right| \\
\leq & \max _{1 \leq i \leq n} \sup _{\gamma}\left|\widehat{G}_{(i)}(\bar{\theta}, \gamma)-G_{(i)}(\bar{\theta}, \gamma)\right|+\max _{1 \leq i \leq n} \sup _{\gamma}\left|G_{(i)}(\bar{\theta}, \gamma)-G_{(i)}\left(\theta_{0}, \gamma\right)\right| \\
\leq & \max _{1 \leq i \leq n} \sup _{(\theta, \gamma)}\left|\widehat{G}_{(i)}(\theta, \gamma)-G_{(i)}(\theta, \gamma)\right|+\max _{1 \leq i \leq n} E\left[M\left(x_{i t}\right)\right] \cdot\left|\bar{\theta}-\theta_{0}\right| .
\end{aligned}
$$

Therefore,

$$
\begin{aligned}
& T \operatorname{Pr}\left[\max _{1 \leq i \leq n} \sup _{\gamma}\left|\widehat{G}_{(i)}(\bar{\theta}, \gamma)-G_{(i)}\left(\theta_{0}, \gamma\right)\right| \geq \eta\right] \\
\leq & T \operatorname{Pr}\left[\max _{1 \leq i \leq n} \sup _{(\theta, \gamma)}\left|\widehat{G}_{(i)}(\theta, \gamma)-G_{(i)}(\theta, \gamma)\right| \geq \frac{\eta}{2}\right] \\
& +T \operatorname{Pr}\left[\left|\bar{\theta}-\theta_{0}\right| \geq \frac{\eta}{2\left(1+\max _{1 \leq i \leq n} E\left[M\left(x_{i t}\right)\right]\right)}\right] \\
= & o(1)
\end{aligned}
$$

by Lemma 13 and Theorem 11 .

We now get back to the proof of Theorem 13. It suffices to prove that

$$
T \operatorname{Pr}\left[\max _{1 \leq i \leq n}\left|\widehat{\gamma}_{i}(\bar{\theta})-\gamma_{i 0}\right| \geq \eta\right]=o(1)
$$

for every $\eta>0$. Let $\eta$ be given, and let $\varepsilon \equiv \inf _{i}\left[G_{(i)}\left(\theta_{0}, \gamma_{i 0}\right)-\sup _{\left\{\gamma_{i}:\left|\gamma_{i}-\gamma_{i 0}\right|>\eta\right\}} G_{(i)}\left(\theta_{0}, \gamma_{i}\right)\right]>0$. Condition on the event

$$
\max _{1 \leq i \leq n} \sup _{\gamma}\left|\widehat{G}_{(i)}(\bar{\theta}, \gamma)-G_{(i)}\left(\theta_{0}, \gamma\right)\right| \leq \frac{1}{3} \varepsilon
$$


which has a probability equal to $1-o\left(\frac{1}{T}\right)$ by $(35)$. We then have

$$
\max _{\left|\gamma_{i}-\gamma_{i 0}\right|>\eta} \widehat{G}_{(i)}\left(\bar{\theta}, \gamma_{i}\right)<\max _{\left|\gamma_{i}-\gamma_{i 0}\right|>\eta} G_{(i)}\left(\theta_{0}, \gamma_{i}\right)+\frac{1}{3} \varepsilon<G_{(i)}\left(\theta_{0}, \gamma_{i 0}\right)-\frac{2}{3} \varepsilon<\widehat{G}_{(i)}\left(\bar{\theta}, \gamma_{i 0}\right)-\frac{1}{3} \varepsilon
$$

This is inconsistent with $\widehat{G}_{(i)}\left(\bar{\theta}, \widehat{\gamma}_{i}(\bar{\theta})\right) \geq \widehat{G}_{(i)}\left(\bar{\theta}, \gamma_{i 0}\right)$, and therefore, $\left|\widehat{\gamma}_{i}(\bar{\theta})-\gamma_{i 0}\right| \leq \eta$ for every $i$.

Corollary $1 \operatorname{Pr}\left[\max _{1 \leq i \leq n}\left|\widehat{\gamma}_{i}(\widetilde{\theta})-\gamma_{i 0}\right| \geq \eta\right]=o\left(T^{-1}\right)$.

Proof. It follows from Theorem 13 above. 


\section{Justification of (26)}

We analyze the asymptotic distribution of

$$
\frac{1}{n T} \sum_{i=1}^{n} \sum_{t=1}^{T} U\left(x_{i t} ; \theta_{0}, \widehat{\gamma}_{i}\left(\theta_{0}\right)\right)
$$

Let $F \equiv\left(F_{1}, \ldots, F_{n}\right)$ denote the collection of (marginal) distribution functions of $x_{i t}$. Let $\widehat{F} \equiv$ $\left(\widehat{F}_{1}, \ldots, \widehat{F}_{n}\right)$, where $\widehat{F}_{i}$ denotes the empirical distribution function for the observation $i$. Define $F(\epsilon) \equiv F+\epsilon \sqrt{T}(\widehat{F}-F)$ for $\epsilon \in\left[0, T^{-1 / 2}\right]$. For each fixed $\theta$ and $\epsilon$, let $\gamma_{i}\left(\theta, F_{i}(\epsilon)\right)$ be the solution to the estimating equation

$$
0=\int V_{i}\left[\theta, \gamma_{i}\left(\theta, F_{i}(\epsilon)\right)\right] d F_{i}(\epsilon),
$$

and let $\mu(F(\epsilon))$ be the solution to the estimating equation

$$
0=\sum_{i=1}^{n} \int\left(U_{i}\left(x_{i t} ; \theta_{0}, \gamma_{i}\left(\theta_{0}, F_{i}(\epsilon)\right)\right)-\mu(F(\epsilon))\right) d F_{i}(\epsilon) .
$$

Note that $\mu(F(0))=0$, and

$$
\begin{aligned}
\mu(\widehat{F}) & \equiv \mu\left(F\left(\frac{1}{\sqrt{T}}\right)\right)=\frac{1}{n} \sum_{i=1}^{n} U_{i}\left(x_{i t} ; \theta_{0}, \gamma_{i}\left(\theta_{0}, F_{i}\left(\frac{1}{\sqrt{T}}\right)\right)\right) \\
& =\frac{1}{n T} \sum_{i=1}^{n} \sum_{t=1}^{T} U\left(x_{i t} ; \theta_{0}, \widehat{\gamma}_{i}\left(\theta_{0}\right)\right) .
\end{aligned}
$$

By a Taylor series expansion, we have

$$
\mu(\widehat{F})-\mu(F)=\frac{1}{\sqrt{T}} \mu^{\epsilon}(0)+\frac{1}{2}\left(\frac{1}{\sqrt{T}}\right)^{2} \mu^{\epsilon \epsilon}(0)+\frac{1}{6}\left(\frac{1}{\sqrt{T}}\right)^{3} \mu^{\epsilon \epsilon \epsilon}(\widetilde{\epsilon})
$$

where $\mu^{\epsilon}(\epsilon) \equiv d \mu(F(\epsilon)) / d \epsilon, \mu^{\epsilon \epsilon}(\epsilon) \equiv d^{2} \mu(F(\epsilon)) / d \epsilon^{2}, \ldots$, and $\widetilde{\epsilon}$ is somewhere in between 0 and $T^{-1 / 2}$. It is shown later in Appendix C.2 that the last term is of order $o_{p}(1)$. We will therefore work with the expansion

$$
\sqrt{n T}(\mu(\widehat{F})-\mu(F))=\sqrt{n T} \frac{1}{\sqrt{T}} \mu^{\epsilon}(0)+\sqrt{n T} \frac{1}{2}\left(\frac{1}{\sqrt{T}}\right)^{2} \mu^{\epsilon \epsilon}(0)+o_{p}(1) .
$$

The expansion (26) follows from combining (38) with (44) and (47) below.

\section{C.1 Details of Expansion (37)}

\section{C.1.1 $\mu^{\epsilon}(0)$}

In order to obtain (44) and (47), we let

$$
h_{i}(\cdot, \epsilon) \equiv U_{i}\left(\cdot ; \theta_{0}, \gamma_{i}\left(\theta_{0}, F_{i}(\epsilon)\right)\right)-\mu(F(\epsilon))
$$


The first order condition may be written as

$$
0=\frac{1}{n} \sum_{i=1}^{n} \int h_{i}(\cdot, \epsilon) d F_{i}(\epsilon)
$$

Differentiating repeatedly with respect to $\epsilon$, we obtain

$$
\begin{aligned}
& 0=\frac{1}{n} \sum_{i=1}^{n} \int \frac{d h_{i}(\cdot, \epsilon)}{d \epsilon} d F_{i}(\epsilon)+\frac{1}{n} \sum_{i=1}^{n} \int h_{i}(\cdot, \epsilon) d \Delta_{i T} \\
& 0=\frac{1}{n} \sum_{i=1}^{n} \int \frac{d^{2} h_{i}(\cdot, \epsilon)}{d \epsilon^{2}} d F_{i}(\epsilon)+2 \frac{1}{n} \sum_{i=1}^{n} \int \frac{d h_{i}(\cdot, \epsilon)}{d \epsilon} d \Delta_{i T} \\
& 0=\frac{1}{n} \sum_{i=1}^{n} \int \frac{d^{3} h_{i}(\cdot, \epsilon)}{d \epsilon^{3}} d F_{i}(\epsilon)+3 \frac{1}{n} \sum_{i=1}^{n} \int \frac{d^{2} h_{i}(\cdot, \epsilon)}{d \epsilon^{2}} d \Delta_{i T}
\end{aligned}
$$

where $\Delta_{i T} \equiv \sqrt{T}\left(\widehat{F}_{i}-F_{i}\right)$.

Equation (41) can be rewritten as

$$
\begin{aligned}
0= & \frac{1}{n} \sum_{i=1}^{n} \int\left(U_{i}^{\gamma_{i}}\left(\cdot ; \theta_{0}, \gamma_{i}\left(\theta_{0}, F_{i}(\epsilon)\right)\right) \gamma_{i}^{\epsilon}\left(\theta_{0}, F_{i}(\epsilon)\right)-\mu^{\epsilon}(F(\epsilon))\right) d F_{i}(\epsilon) \\
& +\frac{1}{n} \sum_{i=1}^{n} \int\left(U_{i}\left(\cdot ; \theta_{0}, \gamma_{i}\left(\theta_{0}, F_{i}(\epsilon)\right)\right)-\mu(F(\epsilon))\right) d \Delta_{i T}
\end{aligned}
$$

Evaluating this expression at $\epsilon=0$, and noting that $E\left[U_{i}^{\gamma_{i}}\right]=0$, we obtain

$$
\mu^{\epsilon}(0)=\frac{1}{n} \sum_{i=1}^{n} \int U_{i} d \Delta_{i T}
$$

\section{C.1.2 $\gamma_{i}^{\epsilon}$}

In the $i$ th observation, $\gamma_{i}\left(\theta_{0}, F_{i}(\epsilon)\right)$ solves the estimating equation

$$
\int V_{i}\left(\cdot ; \theta_{0}, \gamma_{i}\left(\theta_{0}, F_{i}(\epsilon)\right)\right) d F_{i}(\epsilon)=0
$$

Differentiating the LHS with respect to $\epsilon$, we obtain

$$
0=\left(\int \frac{\partial V_{i}(\cdot, \theta, \epsilon)}{\partial \gamma_{i}^{\prime}} d F_{i}(\epsilon)\right) \frac{\partial \gamma_{i}\left(\theta, F_{i}(\epsilon)\right)}{\partial \epsilon}+\int V_{i}(\cdot, \theta, \epsilon) d \Delta_{i T}
$$

Evaluating the expression at $\epsilon=0$, we obtain gives

$$
\gamma_{i}^{\epsilon} \equiv \frac{\partial \gamma_{i}\left(\theta_{0}, F_{i}(0)\right)}{\partial \epsilon}=-\left(E\left[\frac{\partial V_{i}}{\partial \gamma_{i}^{\prime}}\right]\right)^{-1}\left(\frac{1}{\sqrt{T}} \sum_{t=1}^{T} V_{i t}\right) .
$$




\section{C.1.3 $\mu^{\epsilon \epsilon}(0)$}

Equation (42) can be rewritten as

$$
\begin{aligned}
0= & -\frac{1}{n} \sum_{i=1}^{n} \int \mu^{\epsilon \epsilon}(F(\epsilon)) d F_{i}(\epsilon) \\
& +\frac{1}{n} \sum_{i=1}^{n} \int\left(U_{i}^{\gamma_{i} \gamma_{i}}\left(\cdot ; \theta_{0}, \gamma_{i}\left(\theta_{0}, F_{i}(\epsilon)\right)\right)\left(\gamma_{i}^{\epsilon}\left(\theta_{0}, F_{i}(\epsilon)\right) \otimes \gamma_{i}^{\epsilon}\left(\theta_{0}, F_{i}(\epsilon)\right)\right)\right) d F_{i}(\epsilon) \\
& +\frac{1}{n} \sum_{i=1}^{n} \int\left(U_{i}^{\gamma_{i}}\left(\cdot ; \theta_{0}, \gamma_{i}\left(\theta_{0}, F_{i}(\epsilon)\right)\right) \gamma_{i}^{\epsilon \epsilon}\left(\theta_{0}, F_{i}(\epsilon)\right)\right) d F_{i}(\epsilon) \\
& +\frac{2}{n} \sum_{i=1}^{n} \int\left(U_{i}^{\gamma_{i}}\left(\cdot ; \theta_{0}, \gamma_{i}\left(\theta_{0}, F_{i}(\epsilon)\right)\right) \gamma_{i}^{\epsilon}\left(\theta_{0}, F_{i}(\epsilon)\right)-\mu^{\epsilon}(F(\epsilon))\right) d \Delta_{i T}
\end{aligned}
$$

where $U_{i}^{\gamma_{i} \gamma_{i}} \equiv \partial^{2} U_{i} /\left(\partial \gamma_{i} \otimes \partial \gamma_{i}\right)$. Evaluating at $\epsilon=0$, and noting that $E\left[U_{i}^{\gamma_{i}}\right]=0$, we obtain

$$
\begin{aligned}
\mu^{\epsilon \epsilon}(0)= & \frac{1}{n} \sum_{i=1}^{n} E\left[U_{i}^{\gamma_{i} \gamma_{i}}\right]\left(\gamma_{i}^{\epsilon} \otimes \gamma_{i}^{\epsilon}\right)+\frac{2}{n} \sum_{i=1}^{n}\left(\int U_{i}^{\gamma_{i}}\left(\cdot ; \theta_{0}, \gamma_{i 0}\right) d \Delta_{i T}\right) \gamma_{i}^{\epsilon}\left(\theta_{0}, F_{i}(0)\right) \\
= & \frac{1}{n} \sum_{i=1}^{n} E\left[U_{i}^{\gamma_{i} \gamma_{i}}\right]\left(\left(E\left[\frac{\partial V_{i}}{\partial \gamma_{i}^{\prime}}\right]\right)^{-1}\left(\frac{1}{\sqrt{T}} \sum_{t=1}^{T} V_{i t}\right) \otimes\left(E\left[\frac{\partial V_{i}}{\partial \gamma_{i}^{\prime}}\right]\right)^{-1}\left(\frac{1}{\sqrt{T}} \sum_{t=1}^{T} V_{i t}\right)\right) \\
& -\frac{2}{n} \sum_{i=1}^{n}\left(\frac{1}{\sqrt{T}} \sum_{t=1}^{T} U_{i t}^{\gamma_{i}}\right)\left(E\left[\frac{\partial V_{i}}{\partial \gamma_{i}^{\prime}}\right]\right)^{-1}\left(\frac{1}{\sqrt{T}} \sum_{t=1}^{T} V_{i t}\right)
\end{aligned}
$$

or

$$
\begin{aligned}
\mu^{\epsilon \epsilon}(0)= & \frac{1}{n} \sum_{i=1}^{n} E\left[U_{i}^{\gamma_{i} \gamma_{i}}\right]\left[\left(E\left[\frac{\partial V_{i}}{\partial \gamma_{i}^{\prime}}\right]\right)^{-1} \otimes\left(E\left[\frac{\partial V_{i}}{\partial \gamma_{i}^{\prime}}\right]\right)^{-1}\right]\left[\left(\frac{1}{\sqrt{T}} \sum_{t=1}^{T} V_{i t}\right) \otimes\left(\frac{1}{\sqrt{T}} \sum_{t=1}^{T} V_{i t}\right)\right] \\
& -\frac{2}{n} \sum_{i=1}^{n}\left(\frac{1}{\sqrt{T}} \sum_{t=1}^{T} U_{i t}^{\gamma_{i}}\right)\left(E\left[\frac{\partial V_{i}}{\partial \gamma_{i}^{\prime}}\right]\right)^{-1}\left(\frac{1}{\sqrt{T}} \sum_{t=1}^{T} V_{i t}\right)
\end{aligned}
$$

\section{C.1.4 $\gamma_{i}^{\epsilon \epsilon}$}

Second order differentiation of (45) yields

$$
\begin{aligned}
0= & \left(\int \frac{\partial V_{i}(\cdot, \theta, \epsilon)}{\partial \gamma_{i}} d F_{i}(\epsilon)\right) \frac{\partial^{2} \gamma_{i}\left(\theta, F_{i}(\epsilon)\right)}{\partial \epsilon^{2}} \\
& +\left(\int \frac{\partial^{2} V_{i}(\cdot, \theta, \epsilon)}{\partial \gamma_{i} \otimes \partial \gamma_{i}} d F_{i}(\epsilon)\right)\left(\frac{\partial \gamma_{i}\left(\theta, F_{i}(\epsilon)\right)}{\partial \epsilon} \otimes \frac{\partial \gamma_{i}\left(\theta, F_{i}(\epsilon)\right)}{\partial \epsilon}\right) \\
& +2\left(\int \frac{\partial V_{i}(\cdot, \theta, \epsilon)}{\partial \gamma_{i}} d \Delta_{i T}\right) \frac{\partial \gamma_{i}\left(\theta, F_{i}(\epsilon)\right)}{\partial \epsilon} .
\end{aligned}
$$

which characterizes $\gamma_{i}^{\epsilon \epsilon}$. 


\section{C.2 Bounding Remainder Term in (37)}

Lemma 14 below allows us to ignore the last term in equation (37).

\section{Lemma 14}

$$
\begin{aligned}
\operatorname{Pr}\left[\max _{i} \max _{0 \leq \epsilon \leq \frac{1}{\sqrt{T}}}\left|\gamma_{i}^{\epsilon}(\epsilon)\right|>C T^{\frac{1}{10}-v}\right] & =o\left(T^{-1}\right) \\
\operatorname{Pr}\left[\max _{0 \leq \epsilon \leq \frac{1}{\sqrt{T}}}\left|\mu^{\epsilon}(\epsilon)\right|>C T^{\frac{1}{10}}-v\right] & =o\left(T^{-1}\right) \\
\operatorname{Pr}\left[\max _{i} \max _{0 \leq \epsilon \leq \frac{1}{\sqrt{T}}}\left|\gamma_{i}^{\epsilon \epsilon}(\epsilon)\right|>C\left(T^{\frac{1}{10}-v}\right)^{2}\right] & =o\left(T^{-1}\right) \\
\operatorname{Pr}\left[\max _{0 \leq \epsilon \leq \frac{1}{\sqrt{T}}}\left|\mu^{\epsilon \epsilon}(\epsilon)\right|>C\left(T^{\frac{1}{10}}-v\right)^{2}\right] & =o\left(T^{-1}\right) \\
\operatorname{Pr}\left[\max _{i} \max _{0 \leq \epsilon \leq \frac{1}{\sqrt{T}}}\left|\gamma_{i}^{\epsilon \epsilon \epsilon}(\epsilon)\right|>C\left(T^{\frac{1}{10}-v}\right)^{3}\right] & =o\left(T^{-1}\right) \\
\operatorname{Pr}\left[\max _{0 \leq \epsilon \leq \frac{1}{\sqrt{T}}}\left|\mu^{\epsilon \epsilon \epsilon}(\epsilon)\right|>C\left(T^{\frac{1}{10}-v}\right)^{3}\right] & =o\left(T^{-1}\right)
\end{aligned}
$$

for some constant $C>0$ and $0<v<(100 q+120)^{-1}$.

Proof. Proof is almost identical to the argument in Hahn ad Kuersteiner (2004), and so only the last equality is explicitly established here. From (43), we have

$$
0=\frac{1}{n} \sum_{i=1}^{n} \int \frac{d^{3} h_{i}(\cdot, \epsilon)}{d \epsilon^{3}} d F_{i}(\epsilon)+\frac{3}{n} \sum_{i=1}^{n} \int \frac{d^{2} h_{i}(\cdot, \epsilon)}{d \epsilon^{2}} d \Delta_{i T}
$$

where

$$
\begin{aligned}
& \frac{1}{n} \sum_{i=1}^{n} \int \frac{d^{3} h_{i}(\cdot, \epsilon)}{d \epsilon^{3}} d F_{i}(\epsilon) \\
= & -\frac{1}{n} \sum_{i=1}^{n} \int \mu^{\epsilon \epsilon \epsilon}(F(\epsilon)) d F_{i}(\epsilon) \\
& +\frac{1}{n} \sum_{i=1}^{n} \int U_{i}^{\gamma_{i} \gamma_{i} \gamma_{i}}\left(\cdot ; \theta_{0}, \gamma_{i}\left(\theta_{0}, F_{i}(\epsilon)\right)\right)\left(\gamma_{i}^{\epsilon}\left(\theta_{0}, F_{i}(\epsilon)\right) \otimes \gamma_{i}^{\epsilon}\left(\theta_{0}, F_{i}(\epsilon)\right) \otimes \gamma_{i}^{\epsilon}\left(\theta_{0}, F_{i}(\epsilon)\right)\right) d F_{i}(\epsilon) \\
& +\frac{1}{n} \sum_{i=1}^{n} \int\left(U_{i}^{\gamma_{i} \gamma_{i}}\left(\cdot ; \theta_{0}, \gamma_{i}\left(\theta_{0}, F_{i}(\epsilon)\right)\right)\left(\gamma_{i}^{\epsilon \epsilon}\left(\theta_{0}, F_{i}(\epsilon)\right) \otimes \gamma_{i}^{\epsilon}\left(\theta_{0}, F_{i}(\epsilon)\right)\right) d F_{i}(\epsilon)\right. \\
& +\frac{1}{n} \sum_{i=1}^{n} \int\left(U_{i}^{\gamma_{i} \gamma_{i}}\left(\cdot ; \theta_{0}, \gamma_{i}\left(\theta_{0}, F_{i}(\epsilon)\right)\right)\left(\gamma_{i}^{\epsilon \epsilon}\left(\theta_{0}, F_{i}(\epsilon)\right) \otimes \gamma_{i}^{\epsilon \epsilon}\left(\theta_{0}, F_{i}(\epsilon)\right)\right) d F_{i}(\epsilon)\right. \\
& +\frac{1}{n} \sum_{i=1}^{n} \int\left(U_{i}^{\gamma_{i}}\left(\cdot ; \theta_{0}, \gamma_{i}\left(\theta_{0}, F_{i}(\epsilon)\right)\right) \gamma_{i}^{\epsilon \epsilon \epsilon}\left(\theta_{0}, F_{i}(\epsilon)\right)\right) d F_{i}(\epsilon)
\end{aligned}
$$


and

$$
\begin{aligned}
& \frac{3}{n} \sum_{i=1}^{n} \int \frac{d^{2} h_{i}(\cdot, \epsilon)}{d \epsilon^{2}} d \Delta_{i T} \\
= & -\frac{3}{n} \sum_{i=1}^{n} \int \mu^{\epsilon \epsilon}(F(\epsilon)) d \Delta_{i T} \\
& +\frac{3}{n} \sum_{i=1}^{n} \int\left(U_{i}^{\gamma_{i} \gamma_{i}}\left(\cdot ; \theta_{0}, \gamma_{i}\left(\theta_{0}, F_{i}(\epsilon)\right)\right)\left(\gamma_{i}^{\epsilon}\left(\theta_{0}, F_{i}(\epsilon)\right) \otimes \gamma_{i}^{\epsilon}\left(\theta_{0}, F_{i}(\epsilon)\right)\right) d \Delta_{i T}\right. \\
& +\frac{3}{n} \sum_{i=1}^{n} \int\left(U_{i}^{\gamma_{i} \gamma_{i}}\left(\cdot ; \theta_{0}, \gamma_{i}\left(\theta_{0}, F_{i}(\epsilon)\right)\right)\left(\gamma_{i}^{\epsilon}\left(\theta_{0}, F_{i}(\epsilon)\right) \otimes \gamma_{i}^{\epsilon}\left(\theta_{0}, F_{i}(\epsilon)\right)\right) d \Delta_{i T}\right.
\end{aligned}
$$

Combining Lemma 12 in Appendix A and (48)-(52), we can bound $\frac{1}{n} \sum_{i=1}^{n} \int \frac{d^{2} h_{i}(\cdot, \epsilon)}{d \epsilon^{2}} d \Delta_{i T}$ by $C\left(T^{\frac{1}{10}-v}\right)^{3}$ with probability $1-o\left(T^{-1}\right)$. Likewise, using Lemmas 12, and (48)-(52) again, we can conclude that $\frac{1}{n} \sum_{i=1}^{n} \int \frac{d^{3} h_{i}(\cdot, \epsilon)}{d \epsilon^{3}} d F_{i}(\epsilon)$ is equal to $-\mu^{\epsilon \epsilon \epsilon}(F(\epsilon))$ plus terms that can all be bounded by $\frac{1}{n} \sum_{i=1}^{n} \int \frac{d^{2} h_{i}(\cdot, \epsilon)}{d \epsilon^{2}} d \Delta_{i T}$ by $C\left(T^{\frac{1}{10}-v}\right)^{3}$ with probability $1-o\left(T^{-1}\right)$. 


\section{Proof of Theorem 3}

Without loss of generality, we may write

$$
2 B_{n}(\theta)=-\frac{1}{n} \sum_{i=1}^{n} \ln \operatorname{det}\left(\frac{1}{T} H_{i}\left(\theta, \widehat{\gamma}_{i}(\theta)\right)\right)+\frac{1}{n} \sum_{i=1}^{n} \ln \operatorname{det}\left(\frac{1}{T} \Upsilon_{i}\left(\theta, \widehat{\gamma}_{i}(\theta)\right)\right)
$$

We begin with the first component on the RHS of (53). By Assumption 4, each component of $H_{i}\left(\theta, \widehat{\gamma}_{i}(\theta)\right)$ is bounded above by $\sum_{t=1}^{T} M\left(x_{i t}\right)$ such that $\sup _{i} E\left[\left|M\left(x_{i t}\right)\right|^{10 q+12+\delta}\right]<\infty$ for some integer $q \geq(\operatorname{dim}(\theta)+\operatorname{dim}(\gamma)) / 2+2$ and for some $\delta>0$.

Lemma 15 Suppose that $A$ is an $n \times n$ matrix. Then

$$
|\operatorname{det}(A)| \leq n ! \cdot \max \left(\left|a_{i j}\right|\right)^{n}
$$

Proof. By definition, we have

$$
\operatorname{det}(A)=\sum(-1)^{\phi\left(j_{1}, \ldots, j_{n}\right)} \prod_{i=1}^{n} a_{i j_{i}}
$$

where the summation is taken over all permutations $\left(j_{1}, \ldots, j_{n}\right)$ of the set of integers $(1, \ldots, n)$ and $\phi\left(j_{1}, \ldots, j_{n}\right)$ is the number of transpositions required change $(1, \ldots, n)$ into $\left(j_{1}, \ldots, j_{n}\right)$. Because the number of all permutations is equal to $n$ !, we obtain the desired conclusion.

Using Lemma 15, we then obtain that

$$
\ln \operatorname{det}\left(\frac{1}{T} H_{i}\left(\theta, \widehat{\gamma}_{i}(\theta)\right)\right) \leq \ln r !+r \ln \left(\frac{1}{T} \sum_{t=1}^{T} M\left(x_{i t}\right)\right)
$$

where $r=\operatorname{dim}(\gamma)$. It follows that

$$
\left|-\frac{1}{n} \sum_{i=1}^{n} \ln \operatorname{det}\left(\frac{1}{T} H_{i}\left(\theta, \widehat{\gamma}_{i}(\theta)\right)\right)\right| \leq \ln r !+r \frac{1}{n} \sum_{i=1}^{n}\left|\ln \left(\frac{1}{T} \sum_{t=1}^{T} M\left(x_{i t}\right)\right)\right|
$$

By Lemma 2, we have

$$
\operatorname{Pr}\left[\max _{1 \leq i \leq n}\left|\frac{1}{T} \sum_{t=1}^{T}\left(M\left(x_{i t}\right)-E\left[M\left(x_{i t}\right)\right]\right)\right|>\eta\right]=o\left(T^{-1}\right)
$$

from which we obtain ${ }^{12}$

$$
\operatorname{Pr}\left[\max _{1 \leq i \leq n}\left|\ln \left(\frac{1}{T} \sum_{t=1}^{T} M\left(x_{i t}\right)\right)-\ln \left(E\left[M\left(x_{i t}\right)\right]\right)\right|>\eta\right]=o\left(T^{-1}\right)
$$

It follows that

$$
\operatorname{Pr}\left[\left|-\frac{1}{n} \sum_{i=1}^{n} \ln \operatorname{det}\left(\frac{1}{T} H_{i}\left(\theta, \widehat{\gamma}_{i}(\theta)\right)\right)\right|>\ln r !+r \frac{1}{n} \sum_{i=1}^{n} \ln \left(E\left[M\left(x_{i t}\right)\right]\right)+\eta\right]=o\left(T^{-1}\right)
$$

\footnotetext{
${ }^{12}$ In addition to the Condition 4, we need to impose that the minimum of $E\left[M\left(x_{i t}\right)\right]$ is bounded away from zero to make this inequality valid.
} 
from which we conclude that

$$
\operatorname{Pr}\left[\frac{1}{T}\left|-\frac{1}{n} \sum_{i=1}^{n} \ln \operatorname{det}\left(\frac{1}{T} H_{i}\left(\theta, \widehat{\gamma}_{i}(\theta)\right)\right)\right|>\eta\right]=o\left(T^{-1}\right)
$$

for all $\eta>0$.

We now take care of the second component on the RHS of (53). By Assumption 4, each component of $\Upsilon_{i}\left(\theta, \widehat{\gamma}_{i}(\theta)\right)$ is bounded above by $\sum_{l=-m}^{m} w_{T, l}\left(\sum_{t=\max (1, l+1)}^{\min (T, T+l)} M\left(x_{i t}\right) M\left(x_{i t-l}\right)\right)$. Using Lemma 15, we can then conclude that

$$
\ln \operatorname{det}\left(\frac{1}{T} \Upsilon_{i}\left(\theta, \widehat{\gamma}_{i}(\theta)\right)\right) \leq \ln r !+r \ln \left(\frac{1}{T} \sum_{l=-m}^{m} \sum_{t=\max (1, l+1)}^{\min (T, T+l)} M\left(x_{i t}\right) M\left(x_{i t-l}\right)\right)
$$

Using Lemma 2 again, we have

$$
\operatorname{Pr}\left[\max _{1 \leq i \leq n}\left|\frac{1}{T} \sum_{t=\max (1, l+1)}^{\min (T, T+l)}\left(M\left(x_{i t}\right) M\left(x_{i t-l}\right)-E\left[M\left(x_{i t}\right) M\left(x_{i t-l}\right)\right]\right)\right|>\eta\right]=o\left(T^{-1}\right)
$$

and we obtain

$\operatorname{Pr}\left[\max _{1 \leq i \leq n}\left|\ln \left(\frac{1}{T} \sum_{l=-m}^{m} \sum_{t=\max (1, l+1)}^{\min (T, T+l)} M\left(x_{i t}\right) M\left(x_{i t-l}\right)\right)-\ln \left(\sum_{l=-m}^{m} E\left[M\left(x_{i t}\right) M\left(x_{i t-l}\right)\right]\right)\right|>m \eta\right]=o\left(T^{-1}\right.$,

It follows that

$$
\operatorname{Pr}\left[\ln \operatorname{det}\left(\frac{1}{T} \Upsilon_{i}\left(\theta, \widehat{\gamma}_{i}(\theta)\right)\right)>\ln r !+r \frac{1}{n} \sum_{i=1}^{n} \sum_{l=-m}^{m} E\left[M\left(x_{i t}\right) M\left(x_{i t-l}\right)\right]+m \eta\right]=o\left(T^{-1}\right)
$$

Because $E\left[M\left(x_{i t}\right) M\left(x_{i t-l}\right)\right] \leq \sqrt{E\left[M\left(x_{i t}\right)^{2}\right] E\left[M\left(x_{i t-l}\right)^{2}\right]}=E\left[M\left(x_{i t}\right)^{2}\right]$, we have

$$
\operatorname{Pr}\left[\ln \operatorname{det}\left(\frac{1}{T} \Upsilon_{i}\left(\theta, \widehat{\gamma}_{i}(\theta)\right)\right)>\ln r !+2 m \cdot r \frac{1}{n} \sum_{i=1}^{n} E\left[M\left(x_{i t}\right)^{2}\right]+m \eta\right]=o\left(T^{-1}\right)
$$

or

$$
\operatorname{Pr}\left[\frac{1}{T} \ln \operatorname{det}\left(\frac{1}{T} \Upsilon_{i}\left(\theta, \widehat{\gamma}_{i}(\theta)\right)\right)>\frac{\ln r !}{T}+\frac{2 m}{T} r \sup _{i} E\left[M\left(x_{i t}\right)^{2}\right]+\frac{m}{T} \eta\right]=o\left(T^{-1}\right)
$$

Therefore, we obtain

$$
\operatorname{Pr}\left[\frac{1}{T} \ln \operatorname{det}\left(\frac{1}{T} \Upsilon_{i}\left(\theta, \widehat{\gamma}_{i}(\theta)\right)\right)>\eta\right]=o\left(T^{-1}\right)
$$

for all $\eta>0$. 


\section{E Proof of Theorem 4}

We can verify by inspection that $\frac{\partial S_{n}(\theta)}{\partial \theta}$ can be expressed as a sum of terms, all of which are cross section averages of some smooth functions of the form

$$
\begin{aligned}
& \frac{1}{T} \sum_{t=1}^{T} D^{v} \psi\left(x_{i t}, \theta, \widehat{\gamma}_{i}(\theta)\right), \quad \frac{1}{T} \sum_{l=-m}^{m} w_{T, l} \sum_{t=\max (1, l+1)}^{\min (T, T+l)} \frac{\partial \psi\left(x_{i t}, \theta, \widehat{\gamma}_{i}(\theta)\right)}{\partial \gamma^{\prime}} \otimes D^{v} \psi\left(x_{i t-l}, \theta, \widehat{\gamma}_{i}(\theta)\right), \\
& \left(\frac{1}{T} \sum_{t=1}^{T} \frac{\partial^{2} \psi\left(x_{i t}, \theta, \widehat{\gamma}_{i}(\theta)\right)}{\partial \gamma \partial \gamma^{\prime}}\right)^{-1}, \quad\left(\frac{1}{T} \sum_{l=-m}^{m} w_{T, l} \sum_{t=\max (1, l+1)}^{\min (T, T+l)} \frac{\partial \psi\left(x_{i t}, \theta, \widehat{\gamma}_{i}(\theta)\right)}{\partial \gamma} \frac{\partial \psi\left(x_{i t-l}, \theta, \widehat{\gamma}_{i}(\theta)\right)}{\partial \gamma^{\prime}}\right)^{-1}
\end{aligned}
$$

with $|v| \leq 4$. Here, $\phi \equiv(\theta, \gamma)$, and $D^{v} \psi\left(x_{i t}, \phi\right) \equiv \partial^{|\nu|} \psi\left(x_{i t}, \phi\right) /\left(\partial \phi_{1}^{v_{1}} \ldots \partial \phi_{k}^{\nu_{k}}\right)$, where $\nu=\left(\nu_{1}, \ldots, \nu_{k}\right)$ be a vector of non-negative integers $v_{i}$, and $|v|=\sum_{j=1}^{k} v_{j}$. By Assumptions 4, 6, and Lemma 5, we can see that all these terms are $O_{p}(1)$ uniformly over $i$ and $\theta$. 


\section{F Proof of Theorem 6}

Because of the result in the previous section, we only need to consider $\Upsilon_{i}\left(\theta, \widehat{\gamma}_{i}(\theta)\right)$. By Assumption 4, each component of $\Upsilon_{i}\left(\theta, \widehat{\gamma}_{i}(\theta)\right)$ is bounded above by $\sum_{l=-m}^{m} E_{\widehat{\theta}, \widehat{\gamma}_{i}}\left[M\left(x_{i t}\right) M\left(x_{i t-l}\right)\right]$. By Assumption 8 , we have

$$
\sup \sum_{l=-m}^{m} E_{\widehat{\theta}, \widehat{\gamma}_{i}}\left[M\left(x_{i t}\right) M\left(x_{i t-l}\right)\right] \leq 2 m K
$$

where $K=\sup _{(\theta, \gamma) \in \Phi} \sup _{l} E_{\theta, \gamma}\left[M\left(x_{i t}\right) M\left(x_{i t-l}\right)\right]$, and

$$
\ln \operatorname{det}\left(\Upsilon_{i}\left(\theta, \widehat{\gamma}_{i}(\theta)\right)\right) \leq \ln r !+2 r K \ln m
$$

It follows that

$$
\operatorname{Pr}\left[\ln \operatorname{det}\left(\Upsilon_{i}\left(\theta, \widehat{\gamma}_{i}(\theta)\right)\right)>\ln r !+2 r K \ln m+\eta\right]=o\left(T^{-1}\right)
$$

Therefore, we obtain

$$
\operatorname{Pr}\left[\frac{1}{T} \ln \operatorname{det}\left(\frac{1}{T} \Upsilon_{i}\left(\theta, \widehat{\gamma}_{i}(\theta)\right)\right)>\eta\right]=o\left(T^{-1}\right)
$$

for all $\eta>0$ as long as $\frac{\ln m}{T}=o(1)$.

We note that all the above results hold even when the preliminary estimates $\left(\widehat{\theta}, \widehat{\gamma}_{i}\right)$ are replaced by some $\left(\theta^{*}, \gamma_{i}^{*}\right)$. 


\section{G Proof of Theorem 8}

By differentiating $B_{n}$, we obtain that

$$
S_{n}\left(\theta_{0}\right)=[2]+[3]+[4]^{\prime}+[5]^{\prime}
$$

where

$$
\begin{aligned}
{[2]=- } & \frac{1}{2} \frac{1}{n} \sum_{i=1}^{n}\left(\frac{1}{T} \sum_{t=1}^{T} \frac{\partial^{3} \psi_{i t}}{\partial \theta\left(\partial \gamma^{\prime} \otimes \partial \gamma^{\prime}\right)}\right) \operatorname{vec}\left(\left(\frac{1}{T} \sum_{t=1}^{T} \frac{\partial^{2} \psi_{i t}}{\partial \gamma \partial \gamma^{\prime}}\right)^{-1}\right) \\
{[3]=- } & \frac{1}{2} \frac{1}{n} \sum_{i=1}^{n} \frac{\partial \widehat{\gamma}_{i}^{\prime}(\theta)}{\partial \theta}\left(\frac{1}{T} \sum_{t=1}^{T} \frac{\partial^{3} \psi_{i t}}{\partial \gamma\left(\partial \gamma^{\prime} \otimes \partial \gamma^{\prime}\right)}\right) \operatorname{vec}\left(\left(\frac{1}{T} \sum_{t=1}^{T} \frac{\partial^{2} \psi_{i t}}{\partial \gamma \partial \gamma^{\prime}}\right)^{-1}\right) \\
{[4]^{\prime}=} & \frac{1}{2} \frac{1}{n} \sum_{i=1}^{n}\left[\sum_{l=-m}^{m} w_{T, l} E_{\widehat{\theta}, \widehat{\gamma}_{i}}\left[\frac{\partial}{\partial \theta}\left(\left(\frac{\partial \psi_{i t}}{\partial \gamma^{\prime}}\right) \otimes\left(\frac{\partial \psi_{i t-l}}{\partial \gamma^{\prime}}\right)\right)\right]\right] \\
& \cdot \operatorname{vec}\left(\left(\sum_{l=-m}^{m} w_{T, l} E_{\widehat{\theta}, \widehat{\gamma}_{i}}\left[\frac{\partial \psi_{i t}}{\partial \gamma} \frac{\partial \psi_{i t-l}}{\partial \gamma^{\prime}}\right]\right)^{-1}\right)
\end{aligned}
$$

and

$$
\begin{aligned}
{[5]^{\prime}=} & \frac{1}{2} \frac{1}{n} \sum_{i=1}^{n} \frac{\partial \widehat{\gamma}_{i}^{\prime}(\theta)}{\partial \theta}\left[\sum_{l=-m}^{m} w_{T, l} E_{\widehat{\theta}, \widehat{\gamma}_{i}}\left[\frac{\partial}{\partial \gamma}\left(\left(\frac{\partial \psi_{i t}}{\partial \gamma^{\prime}}\right) \otimes\left(\frac{\partial \psi_{i t}}{\partial \gamma^{\prime}}\right)\right)\right]\right] \\
& \cdot \operatorname{vec}\left(\left(\sum_{l=-m}^{m} w_{T, l} E_{\widehat{\theta}, \widehat{\gamma}_{i}}\left[\frac{\partial \psi_{i t}}{\partial \gamma} \frac{\partial \psi_{i t}}{\partial \gamma^{\prime}}\right]\right)^{-1}\right)
\end{aligned}
$$

We can see that [2] and [3] are identical to the ones in the previous section. Because we have already established

$$
[2]+[3]=-\frac{1}{2} \frac{1}{n} \sum_{i=1}^{n} E\left[U_{i t}^{\gamma \gamma}\right] \operatorname{vec}\left(\left(E\left[V_{i t}^{\gamma}\right]\right)^{-1}\right)+o_{p}(1)
$$

we will focus on $[4]^{\prime}$ and $[5]^{\prime}$ here.

Because

$$
\begin{aligned}
\frac{\partial}{\partial \theta}\left(\left(\frac{\partial \psi_{i t}(\theta, \gamma)}{\partial \gamma^{\prime}}\right) \otimes\left(\frac{\partial \psi_{i t-l}(\theta, \gamma)}{\partial \gamma^{\prime}}\right)\right) & =\left(U_{i t}^{\gamma}+\rho_{i} V_{i t}^{\gamma}\right) \otimes V_{i t-l}^{\prime}+V_{i t}^{\prime} \otimes\left(U_{i t-l}^{\gamma}+\rho_{i} V_{i t-l}^{\gamma}\right) \\
\frac{\partial}{\partial \gamma}\left(\left(\frac{\partial \psi_{i t}(\theta, \gamma)}{\partial \gamma^{\prime}}\right) \otimes\left(\frac{\partial \psi_{i t}(\theta, \gamma)}{\partial \gamma^{\prime}}\right)\right) & =V_{i t}^{\gamma} \otimes V_{i t-l}^{\prime}+V_{i t}^{\prime} \otimes V_{i t-l}^{\gamma}
\end{aligned}
$$

and

$$
\frac{\partial \widehat{\gamma}_{i}^{\prime}(\theta)}{\partial \theta}=-\rho_{i}+o_{p}(1)
$$


we can write

$$
\begin{aligned}
{[4]^{\prime}+[5]^{\prime}=} & \frac{1}{2} \frac{1}{n} \sum_{i=1}^{n}\left[\sum_{l=-m}^{m} w_{T, l} E_{\widehat{\theta}, \widehat{\gamma}_{i}}\left[\begin{array}{c}
U_{i t}^{\gamma}\left(\theta_{0}, \widehat{\gamma}_{i}\left(\theta_{0}\right)\right) \otimes V_{i t-l}\left(\theta_{0}, \widehat{\gamma}_{i}\left(\theta_{0}\right)\right)^{\prime} \\
+V_{i t}\left(\theta_{0}, \widehat{\gamma}_{i}\left(\theta_{0}\right)\right)^{\prime} \otimes U_{i t-l}^{\gamma}\left(\theta_{0}, \widehat{\gamma}_{i}\left(\theta_{0}\right)\right)
\end{array}\right]\right] \\
& \cdot \operatorname{vec}\left(\left(\sum_{l=-m}^{m} w_{T, l} E_{\widehat{\theta}, \widehat{\gamma}_{i}}\left[V_{i t}\left(\theta_{0}, \widehat{\gamma}_{i}\left(\theta_{0}\right)\right) V_{i t-l}\left(\theta_{0}, \widehat{\gamma}_{i}\left(\theta_{0}\right)\right)^{\prime}\right]\right)^{-1}\right)+o_{p}(1)
\end{aligned}
$$

Using Lemma 9, we obtain

$$
\max _{i}\left|\sum_{l=-m}^{m} w_{T, l} E_{\widehat{\theta}, \widehat{\gamma}_{i}}\left[V_{i t}\left(\theta_{0}, \widehat{\gamma}_{i}\left(\theta_{0}\right)\right) V_{i t-l}\left(\theta_{0}, \widehat{\gamma}_{i}\left(\theta_{0}\right)\right)^{\prime}\right]-\sum_{l=-\infty}^{\infty} E\left[V_{i t} V_{i t-l}^{\prime}\right]\right|=o_{p}(1)
$$

Furthermore, if the conditional likelihood is properly defined, then we should have $V_{i t}$ serially uncorrelated, which implies that

$$
\begin{aligned}
\max _{i}\left|\sum_{l=-m}^{m} w_{T, l} E_{\widehat{\theta}_{,}, \hat{\gamma}_{i}}\left[V_{i t}\left(\theta_{0}, \widehat{\gamma}_{i}\left(\theta_{0}\right)\right) V_{i t-l}\left(\theta_{0}, \widehat{\gamma}_{i}\left(\theta_{0}\right)\right)^{\prime}\right]-E\left[V_{i t} V_{i t}^{\prime}\right]\right| \\
=\max _{i}\left|\sum_{l=-m}^{m} w_{T, l} E_{\widehat{\theta}, \widehat{\gamma}_{i}}\left[V_{i t}\left(\theta_{0}, \widehat{\gamma}_{i}\left(\theta_{0}\right)\right) V_{i t-l}\left(\theta_{0}, \widehat{\gamma}_{i}\left(\theta_{0}\right)\right)^{\prime}\right]+E\left[V_{i t}^{\gamma}\right]\right|=o_{p}(1)
\end{aligned}
$$

where the first equality is based on the information equality. Therefore, we obtain

$$
\begin{aligned}
& {[4]^{\prime}+[5]^{\prime} } \\
= & -\frac{1}{2} \frac{1}{n} \sum_{i=1}^{n}\left[\sum_{l=-m}^{m} w_{T, l} E_{\widehat{\theta}, \widehat{\gamma}_{i}}\left(\begin{array}{c}
U_{i t}^{\gamma}\left(\theta_{0}, \widehat{\gamma}_{i}\left(\theta_{0}\right)\right) \otimes V_{i t-l}\left(\theta_{0}, \widehat{\gamma}_{i}\left(\theta_{0}\right)\right)^{\prime} \\
+V_{i t}\left(\theta_{0}, \widehat{\gamma}_{i}\left(\theta_{0}\right)\right)^{\prime} \otimes U_{i t-l}^{\gamma}\left(\theta_{0}, \widehat{\gamma}_{i}\left(\theta_{0}\right)\right)
\end{array}\right)\right] \cdot \operatorname{vec}\left(E\left[V_{i t}^{\gamma}\right]^{-1}\right)+o_{p}(1)
\end{aligned}
$$

Using Lemma 9 again, we obtain

$$
[4]^{\prime}+[5]^{\prime}=-\frac{1}{2} \frac{1}{n} \sum_{i=1}^{n} \sum_{l=-\infty}^{\infty} E\left[U_{i t}^{\gamma} \otimes V_{i t-l}^{\prime}+V_{i t}^{\prime} \otimes U_{i t-l}^{\gamma}\right] \operatorname{vec}\left(E\left[V_{i t}^{\gamma}\right]^{-1}\right)+o_{p}(1)
$$

Because we have ${ }^{13}$

$$
\begin{aligned}
& \left(U_{i t}^{\gamma} \otimes V_{i t-l}^{\prime}\right) \operatorname{vec}\left(E\left[V_{i t}^{\gamma}\right]^{-1}\right)=U_{i t}^{\gamma} E\left[V_{i t}^{\gamma}\right]^{-1} V_{i t-l}=-U_{i t}^{\gamma} \widetilde{V}_{i t-l} \\
& \left(V_{i t}^{\prime} \otimes U_{i t-l}^{\gamma}\right) \operatorname{vec}\left(E\left[V_{i t}^{\gamma}\right]^{-1}\right)=U_{i t-l}^{\gamma} E\left[V_{i t}^{\gamma}\right]^{-1} V_{i t}=-U_{i t-l}^{\gamma} \widetilde{V}_{i t}
\end{aligned}
$$

it follows that

$$
\begin{aligned}
{[4]^{\prime}+[5]^{\prime} } & =\frac{1}{2} \frac{1}{n} \sum_{i=1}^{n} \sum_{l=-\infty}^{\infty} E\left[U_{i t}^{\gamma} \widetilde{V}_{i t-l}+U_{i t-l}^{\gamma} \widetilde{V}_{i t}\right]+o_{p}(1) \\
& =\frac{1}{n} \sum_{i=1}^{n} \sum_{l=-\infty}^{\infty} E\left[U_{i t}^{\gamma} \widetilde{V}_{i t-l}\right]+o_{p}(1)
\end{aligned}
$$

We note that, because of Lemma 10, all the above results hold even when the preliminary estimates $\left(\widehat{\theta}, \widehat{\gamma}_{i}\right)$ are replaced by some $\left(\theta^{*}, \gamma_{i}^{*}\right)$ as long as $\left\|\theta^{*}-\theta_{0}\right\|=O_{p}\left(T^{-2 / 5}\right)$ and $\sup _{i}\left\|\gamma_{i}^{*}-\gamma_{i 0}\right\|=$ $O_{p}\left(T^{-2 / 5}\right)$.

\footnotetext{
${ }^{13}$ See, e.g., Magnus \& Neudecker (1988, p. 31, eq. (3)).
} 


\section{References}

[1] Hahn, J., And G. Kuersteiner (2004): "Bias Reduction for Dynamic Nonlinear Panel Models with Fixed Effects", unpublished manuscript.

[2] Hall, P. And C. Heyde (1980): Martingale Limit Theory and its Application, Academic Press, New York.

[3] LahiRi, S. (1992): "Edgeworth Correction by Moving Block Bootstrap for Stationary and Nonstationary Data," in Exploring the Limits of Bootstrap, ed. by R. LePage, and L. Billard, pp. 183-214. Wiley.

[4] Magnus, J. And H. Neudecker (1988): Matrix Differential Calculus with Applications in Statistics and Econometrics, Wiley. 


\section{CEMFI WORKING PAPERS}

0401 Andres Almazan, Javier Suarez and Sheridan Titman: "Stakeholders, transparency and capital structure".

0402 Antonio Diez de los Rios: "Exchange rate regimes, globalisation and the cost of capital in emerging markets".

0403 Juan J. Dolado and Vanessa Llorens: "Gender wage gaps by education in Spain: Glass floors vs. glass ceilings".

0404 Sascha O. Becker, Samuel Bentolila, Ana Fernandes and Andrea Ichino: "Job insecurity and children's emancipation".

0405 Claudio Michelacci and David Lopez-Salido: "Technology shocks and job flows".

0406 Samuel Bentolila, Claudio Michelacci and Javier Suarez. "Social contacts and occupational choice".

0407 David A. Marshall and Edward Simpson Prescott. "State-contingent bank regulation with unobserved actions and unobserved characteristics".

0408 Ana Fernandes: "Knowledge, technology adoption and financial innovation".

0409 Enrique Sentana, Giorgio Calzolari and Gabriele Fiorentini: "Indirect estimation of conditionally heteroskedastic factor models".

0410 Francisco Peñaranda and Enrique Sentana: "Spanning tests in return and stochastic discount factor mean-variance frontiers: A unifying approach".

0411 F. Javier Mencía and Enrique Sentana: "Estimation and testing of dynamic models with generalised hyperbolic innovations".

0412 Edward Simpson Prescott. "Auditing and bank capital regulation".

0413 Víctor Aguirregabiria and Pedro Mira: "Sequential estimation of dynamic discrete games".

0414 Kai-Uwe Kühn and Matilde Machado: "Bilateral market power and vertical integration in the Spanish electricity spot market".

0415 Guillermo Caruana, Liran Einav and Danie/ Quint. "Multilateral bargaining with concession costs".

0416 David S. Evans and A. Jorge Padilla: "Excessive prices: Using economics to define administrable legal rules".

0417 David S. Evans and A. Jorge Padilla: "Designing antitrust rules for assessing unilateral practices: A neo-Chicago approach".

0418 Rafael Repullo: "Policies for banking crises: A theoretical framework".

0419 Francisco Peñaranda: "Are vector autoregressions an accurate model for dynamic asset allocation?"

0420 Ángel León and Diego Piñeiro: "Valuation of a biotech company: A real options approach".

0421 Javier A/varez and Manuel Arellano: "Robust likelihood estimation of dynamic panel data models".

0422 Abel Elizalde and Rafael Repullo: "Economic and regulatory capital. What is the difference?".

0501 Claudio Michelacci and Vincenzo Quadrini: "Borrowing from employees: Wage dynamics with financial constraints".

0502 Gerard Llobet and Javier Suarez. "Financing and the protection of innovators". 
0503 Juan-José Ganuza and José S. Penalva: "On information and competition in private value auctions".

0504 Rafael Repullo: "Liquidity, risk-taking, and the lender of last resort".

0505 Marta González and Josep Pijoan-Mas: "The flat tax reform: A general equilibrium evaluation for Spain".

0506 Claudio Michelacci and Olmo Silva: "Why so many local entrepreneurs?".

0507 Manuel Arellano and Jinyong Hahn: "Understanding bias in nonlinear panel models: Some recent developments".

0508 Aleix Calveras, Juan-José Ganuza and Gerard Llobet. "Regulation and opportunism: How much activism do we need?".

0509 Ángel León, Javier Mencía and Enrique Sentana: "Parametric properties of semi-nonparametric distributions, with applications to option valuation".

0601 Beatriz Domínguez, Juan José Ganuza and Gerard Llobet. "R\&D in the pharmaceutical industry: a world of small innovations".

0602 Guillermo Caruana and Liran Einav. "Production targets".

0603 Jose Ceron and Javier Suarez. "Hot and cold housing markets: International evidence".

0604 Gerard Llobet and Michael Manove: "Network size and network capture".

0605 Abel Elizalde: "Credit risk models I: Default correlation in intensity models".

0606 Abel Elizalde: "Credit risk models II: Structural models".

0607 Abel Elizalde: "Credit risk models III: Reconciliation reduced - structural models".

0608 Abel Elizalde: "Credit risk models IV: Understanding and pricing CDOs".

0609 Gema Zamarro: "Accounting for heterogeneous returns in sequential schooling decisions".

0610 Max Bruche: "Estimating structural models of corporate bond prices".

0611 Javier Díaz-Giménez and Josep Pijoan-Mas: "Flat tax reforms in the U.S.: A boon for the income poor".

0612 Max Bruche and Carlos González-Aguado: "Recovery rates, default probabilities and the credit cycle".

0613 Manuel Arellano and Jinyong Hahn: "A likelihood-based approximate solution to the incidental parameter problem in dynamic nonlinear models with multiple effects". 